\title{
Two Dimensional Finite-Difference Time-Domain Formulation for Sound Propagation in A Temperature-Dependent Elastomer-Fluid Medium
}

\author{
Yunke Huang, ${ }^{1, a)}$ Hong Hou, ${ }^{1, b)}$ Selda Oterkus, ${ }^{2}$ Zhengyu Wei, ${ }^{1}$ and Nansha Gao ${ }^{1}$ \\ ${ }^{1}$ Department of Environmental Engineering, School of marine science and technology, Northwestern \\ Polytechnical University, 127 West Youyi Road, Beilin District, Xi'an, Shaanxi,710072, P.R.China \\ ${ }^{2}$ Department of Naval Architecture, Ocean \& Marine Engineering, University of Strathclyde, 100 \\ Montrose Street, Glasgow, G4 OLZ, UK
}

\begin{abstract}
This study focuses on the two dimensional finite-difference time-domain (FDTD) formulations to investigate the acoustic wave propagation in elastomers contained in fluid region under different thermal conditions. The developed FDTD formulation is based on direct solution of the time-domain wave equation and the Havriliak-Negami $(\mathrm{H}-\mathrm{N})$ dynamic mechanical response of the elastomers. The H-N representation including double fractional derivative operators can be accurately transferred from the frequency-domain to the timedomain by using Riemann-Liouville theory and the Grunwald-Letnikov operator for fractional derivative approximations. Since the Williams-Landel-Ferry (WLF) shift function is related to the relaxation time for different thermal conditions, the proposed scheme represents a simple and accurate prediction of acoustic wave propagation for varying thermal conditions. The pulse-wave propagation in viscous fluid field is simulated by investigating the Navier-Stokes equations. The acoustic properties of different elastomers in a variety of temperatures are obtained by means of the proposed FDTD formulation and validated by a good agreement with the experimental data over a wide frequency range. Additionally, the 2D examples relevant to wave propagation in different elastomers contained in a fluid field are implemented. The proposed FDTD formulation can be used to predict 2-D acoustic wave propagation in different thermal conditions accurately.
\end{abstract}

Keywords: FDTD formulation, Wave propagation, H-N dispersion, Temperature effect

\section{Introduction}

Recently researches on the interaction between acoustic wave and elastomers have received increasing attention due to the promising applications in the area of underwater acoustic insulation. ${ }^{1}$ Acoustic pulses have been developed to determine the acoustic properties of elastomers contained in fluid field, since their capability to induce significant excitation for particle motion of both fluid flow and elastomer. ${ }^{2}$ As for large internal friction and good damping characteristics, the thermal effects lead to the change of molecular mechanisms and sound absorption performance of elastomers. Hence the acoustic pulse

\footnotetext{
a) Also at: Department of Naval Architecture, Ocean \& Marine Engineering, University of Strathclyde, 100 Montrose Street, Glasgow, G4 OLZ, UK

b) Electronic mail: houhong@ nwpu.edu.cn
} 
propagating in elastomers and its application in fluid field in different thermal environments need to be investigated.

The acoustic fields excited in the elastomers mainly depend on the mechanical response of the media, but the lack of data and accurate analytical models has been an obstacle for both theoretical and experimental studies. ${ }^{3}$ The common properties of fractal physiological structure and anomalies of dynamic mechanisms produce a strong dispersion for mechanical susceptibility. ${ }^{4}$ The associated hysteresis yields a wide spectrum of relaxation times. Thus the accurate representation of the experimental mechanical response in frequency domain cannot be described by a simple exponential expression with a single relaxation. ${ }^{5}$ A number of empirical models including Maxwell, Cole-Cole (C-C) and Havriliak-Negami (H-N) relations have been proposed to fit the dynamic mechanical spectra. ${ }^{6-10}$ In particular, $\mathrm{H}-\mathrm{N}$ relation provides an extensible frequency-domain model to parametrize the mechanical response of dispersive media and a better description for the asymmetric relaxation loss peak, especially under acoustic pulse excitation. Effects of temperature on dynamic mechanical parameters can be considered by H-N relations. ${ }^{11,12}$

The FDTD algorithm has been validated as one of the powerful computational methods for wave propagation modelling in complex media, such as biological, electromagnetic ${ }^{4,5,11}$ and porous materials. ${ }^{14-16}$ The approximation of acoustic wave propagation in $\mathrm{H}-\mathrm{N}$ dispersive media by using FDTD algorithm contains the transformation of the $\mathrm{H}-\mathrm{N}$ expression from the frequency-domain into the time-domain. Since the frequency-domain representation of $\mathrm{H}-\mathrm{N}$ complex modulus exhibits the fractional powers of the angular frequency, the evaluation of fractional derivatives based on finite difference faces mathematical challenges ${ }^{5}$. The $\mathrm{Z}$ transform is a common approach by reducing the time-domain convolution integral to numerical multiplication. ${ }^{17,18}$ An auxiliary differential equation can also be formulated for the FDTD simulation by using rational or polynomial functions to the evaluate complex modulus. ${ }^{19,20}$ The application of Riemann-Liouville theory involves additional ordinary differential equations to analyse fractional derivatives. ${ }^{21}$ However the double fractional derivative operator relevant to the $\mathrm{H}-\mathrm{N}$ mechanical response is directly incorporated in FDTD scheme by using the above method, resulting in the inaccuracy of wave propagation simulation. ${ }^{4}$

The application of $\mathrm{H}-\mathrm{N}$ dispersive media in underwater conditions requires coupling with the fluid field. Acoustic wave propagation in the fluid field has been investigated by using both theoretical and computational methods. ${ }^{22}$ From the perspective of numerical simulation, modelling of finite-amplitude sound propagation in a homogenous fluid has been proposed by using the FDTD method. ${ }^{23}$ The acoustic scattering for one dimensional fluid-fluid interface is modelled by modifying the FDTD scheme to solve coupled differential equations. ${ }^{24}$ For unbounded problems, the concept of perfect-matched-layer is implemented in the FDTD modelling of wave propagating in a fluid field, leading to the achievement of the optimum absorbing boundary condition. ${ }^{25}$

In this study, a two-dimensional FDTD scheme for elastomers within a fluid field is presented, considering the thermal effects on both elastomer and viscous fluid. The acoustic wave propagation in elastomers is suggested in more realistic underwater conditions. The FDTD formulation for elastomers with the $\mathrm{H}-\mathrm{N}$ dispersive mechanical response is presented based on Riemann-Liouville theory of fractional differentiation. The fractional derivative is 
approximated by using the Grunwald-Letnikov operator. The thermal effect on elastomers is induced by transfer factor of relaxation time, which can be calculated by the WLF equation in different temperatures. Additionally, the FDTD expression for ideal quasi-static fluid is derived by using the Navier-Stokes equations with sound velocity dependent to the temperature. The sound absorption properties for two H-N type elastomers at different temperatures are calculated by using proposed FDTD algorithm, validating the algorithm by the good agreement with the experimental data. The two-dimensional spatial distributions of sound pressure and particle velocities excited by an acoustic pulse are further simulated for $\mathrm{H}-\mathrm{N}$ medium at different temperatures, which demonstrate the thermal effect on wave propagation in $\mathrm{H}-\mathrm{N}$ type elastomers.

\section{FDTD formulation for wave propagation equation in $\mathrm{H}-\mathrm{N}$ dispersive media}

\section{A. Constitutive Equation of 2-D elastic wave}

The linear elastic wave is governed by Newton's equation of motion and the generalized Hooke's law. In the two-dimensional Cartesian coordinates $(i, j=1,2)$, the governing equations of elastic wave for $\mathrm{H}-\mathrm{N}$ media can be written as ${ }^{26}$

$$
\begin{gathered}
\rho_{S} \frac{\partial v_{i}}{\partial t}=\frac{\partial \tau_{i j}}{\partial x_{j}} \\
\frac{\partial \tau_{i j}}{\partial t}=C_{i j k l} \frac{\partial v_{k}}{\partial x_{l}}
\end{gathered}
$$

in which $\rho_{S}$ represents the mass density of H-N media, $v_{i}$ represents the particle velocity in each direction, $\tau_{i j}$ represents the component of the applied stress tensor and $C_{i j k l}(i, j, k, l=1,2)$ represents the component of the second-order elasticity tensor. For isotropic $\mathrm{H}-\mathrm{N}$ media the elasticity tensor can be calculated by

$$
C_{i j k l}=\lambda_{S} \delta_{i j} \delta_{k l}+\mu_{S}\left(\delta_{i k} \delta_{j l}+\delta_{i l} \delta_{j k}\right)
$$

in which $\lambda_{S}$ and $\mu_{S}$ represent the first and the second Lame coefficients respectively, and $\delta$ represents the Kronecker delta.

Under the assumption that the acoustic wave propagating in the two-dimensional $\mathrm{H}-\mathrm{N}$ media without shear stress and shear velocity, the stress field can be replaced by the sound pressure field in the propagation direction. The constitutive relations between the particle velocity and the sound pressure can be expressed as

$$
\begin{gathered}
\frac{\partial p_{S}}{\partial t}=\left(C_{1111} \frac{\partial u_{S}}{\partial x}+C_{1122} \frac{\partial v_{S}}{\partial y}\right) \\
\frac{\partial u_{S}}{\partial t}=\frac{1}{\rho_{S}} \frac{\partial p_{S}}{\partial x} \\
\frac{\partial v_{S}}{\partial t}=\frac{1}{\rho_{S}} \frac{\partial p_{S}}{\partial y}
\end{gathered}
$$


in which $p_{S}$ represents the sound pressure, $u_{S}$ represents the particle velocity in the $\mathrm{x}$ direction and $v_{S}$ represents the particle velocity in the y-direction. Eq. (3a) can be rewritten by substituting $C_{1111}=\lambda_{S}+2 \mu_{S}$ and $C_{1122}=\lambda_{S}$ as

$$
\frac{\partial p_{S}}{\partial t}=\left(\lambda_{S}+2 \mu_{S}\right) \frac{\partial u_{S}}{\partial x}+\lambda_{S} \frac{\partial v_{S}}{\partial y}
$$

The Lame coefficients have the relations with the complex Young's modulus as

$$
\begin{aligned}
& \lambda_{S}(\omega)=\frac{E^{*}(\omega)}{2(1-v)} \\
& \mu_{S}(\omega)=\frac{E^{*}(\omega)}{2(1+v)}
\end{aligned}
$$

in which $E^{*}$ represents the complex modulus and $v$ represents the Poisson's ratio of the material. For isotropic Havriliak-Negami dispersive media, the complex Young's modulus in frequency domain is given by ${ }^{27}$

$$
E^{*}(\omega)=E_{\infty}+\frac{E_{0}-E_{\infty}}{\left(1+(i \omega \tau)^{\alpha}\right)^{\beta}}
$$

in which $\alpha, \beta, E_{0}, E_{\infty}$ and $\tau$ are $\mathrm{H}-\mathrm{N}$ parameters to describe mechanical response of the $\mathrm{H}$ $\mathrm{N}$ dispersive material. $E_{0}$ represents the rubbery modulus related to low frequency stage, $E_{\infty}$ represents the glassy modulus related to high frequency stage, $\alpha$ and $\beta$ are positive and adjustable parameters determining the shape and the width of the loss peak, and $\tau$ is the principal relaxation time. The complex modulus can be described by using storage modulus $E^{\prime}$ as real part and loss modulus $E^{\prime \prime}$ as imaginary part ${ }^{27}$

$$
E^{*}=E^{\prime}+i E^{\prime \prime}
$$

which leads to

The loss factor, $\tan \delta$ is introduced to define the relation between storage modulus $E^{\prime}$ and the loss modulus $E^{\prime \prime}$ as

$$
\tan \delta=\frac{E^{\prime \prime}}{E^{\prime}}=\frac{\left(E_{\infty}-E_{0}\right) \sin (\beta \theta)}{E_{\infty}\left[1+2(\omega \tau)^{\alpha} \cos (\alpha \tau / 2)+(\omega \tau)^{2 \alpha}\right]^{\beta / 2}+\left(E_{0}-E_{\infty}\right) \cos (\beta \theta)}
$$

in which the loss angle is represented by $\theta=\arctan \frac{(\omega \tau)^{\alpha} \sin (\alpha \pi / 2)}{1+(\omega \tau)^{\alpha} \cos (\alpha \pi / 2)}$.

Hence Eq. (4) can be rewritten by using the definitions provided in Eqs. (5a), (5b) and (6) as

$$
\frac{\partial p}{\partial t}=\left(E_{\infty}+\frac{E_{0}-E_{\infty}}{\left[1+(i \omega \tau)^{\alpha}\right]^{\beta}}\right)\left(\frac{3-v}{2(1-v)(1+v)} \frac{\partial u}{\partial x}+\frac{1}{2(1-v)} \frac{\partial v}{\partial y}\right)
$$




\section{B. Temperature effect based on improved WLF equation}

The thermal effect on $\mathrm{H}-\mathrm{N}$ mechanical response can be investigated by using WLF (Williams-Landel-Ferry) shift function to modify the relaxation time, $\tau$ as ${ }^{28}$

$$
\log \phi_{T}=\log \left(\frac{\tau}{\tau_{0}}\right)=\frac{-C_{1}\left(T-T_{0}\right)}{C_{2}+\left(T-T_{0}\right)}
$$

in which $\phi_{T}$ is the temperature transfer factor, $T_{0}$ is the reference temperature; $\tau$ and $\tau_{0}$ represent the relaxation times corresponding to $T$ and $T_{0}$ respectively. $C_{1}$ and $C_{2}$ in Eq. (11) are empirical parameters related to thermal expansion coefficient, only depending on the reference temperature. The empirical parameters $C_{1}^{\prime}$ and $C_{2}^{\prime}$ with respect to reference temperature $T_{M}$ can be obtained based on the deviation between glass-transient temperature $T_{0}$ and reference temperature $T_{M}=T_{0}+\Delta T^{28}$

$$
\left\{\begin{array}{l}
C_{1}^{\prime}=\frac{C_{1} C_{2}}{C_{2}+\Delta T} \\
C_{2}^{\prime}=C_{2}+\Delta T
\end{array}\right.
$$

in which $C_{1}^{\prime}, C_{2}^{\prime}$ represent the empirical parameters with respect to the glass-transition temperature $T_{0}$.

According to WLF equation, the transfer factor, $\phi_{T_{M}}$ from reference temperature $T_{M}$ to temperature $T$ can be determined by the following equation

$$
\log \phi_{T_{M}}=\frac{C_{1}^{\prime}\left(T-T_{M}\right)}{C_{2}^{\prime}+\left(T-T_{M}\right)}
$$

Here $C_{1}^{\prime}$ and $C_{2}^{\prime}$ are empirical parameters at temperature $T_{M}$ and can be evaluated by Eq. (10). The relaxation time for temperature $T$ then can be determined as

$$
\tau(T)=\tau\left(T_{M}\right) 10^{-\frac{C_{1}^{\prime}\left(T-T_{M}\right)}{C_{2}^{\prime}+\left(T-T_{M}\right)}}
$$

The H-N mechanical response for the temperature $T$ can be derived based on the mechanical response under the condition $T_{M}$ as

$$
E^{*}(\omega, \tau(T))=E^{*}\left[\omega, \tau\left(T_{M}\right) 10^{-\frac{C_{1}^{\prime}\left(T-T_{M}\right)}{C_{2}^{\prime}+\left(T-T_{M}\right)}}\right]
$$

and the constitutive relation in different temperatures can be expressed as

$$
\frac{\partial p(T)}{\partial t}=\left(E_{\infty}+\frac{E_{0}-E_{\infty}}{\left[1+(i \omega \tau(T))^{\alpha}\right]^{\beta}}\right)\left(\frac{3-v}{2(1-v)(1+v)} \frac{\partial u}{\partial x}+\frac{1}{2(1-v)} \frac{\partial v}{\partial y}\right)
$$

\section{Riemann-Liouville theory for fractional operator approximation}

The angular frequency term with fractional difference in Eq. (16) can be shown as $\left[1+(i \omega \tau)^{\alpha}\right]^{-\beta} \mathbf{J}$, in which $\mathbf{J}=\frac{\partial \mathbf{v}}{\partial x_{i}}$ represents the spatial gradient of the particle velocity and $\mathbf{v}$ represents the particle velocity vector. The transformation form of angular frequency term into time domain can be expressed by fractional differential operators as 


$$
D_{t}^{\alpha, \beta} \mathbf{J}=\left[1+\tau^{\alpha} D_{t}^{\alpha}\right]^{-\beta} \mathbf{J}
$$

where $D_{t}^{\alpha}$ is the $\alpha$-order fractional differential operator. Based on the Riemann-Liouville theory, Eq. (17) follows that ${ }^{29}$

$$
D_{t}^{\alpha, \beta} \mathbf{J}=\frac{1}{\Gamma(\beta)} \int_{0}^{+\infty} u^{\beta-1} e^{-u\left(1+\tau^{\alpha} D_{t}^{\alpha}\right)} \mathbf{J} d u
$$

The Taylor-Series expansion can be used to solve the exponential function as ${ }^{30}$

$$
e^{-u\left(1+\tau^{\alpha} D_{t}^{\alpha}\right)}=\sum_{n=0}^{+\infty} u^{n} \frac{\left(-\tau^{\alpha}\right)^{n}}{n !} D_{t}^{n \alpha}
$$

The integral representation can be considered by using the Gamma function ${ }^{30}$

$$
\Gamma(n+\beta)=\int_{0}^{+\infty} u^{n+\beta-1} e^{-u} d u
$$

Eq. (18) for $D_{t}^{\alpha, \beta} \mathbf{J}$ becomes ${ }^{5}$

$$
\begin{aligned}
D_{t}^{\alpha, \beta} \mathbf{J} & =\frac{1}{\Gamma(\beta)} \int_{0}^{+\infty} u^{\beta-1}\left(\sum_{n=0}^{+\infty} u^{n} \frac{\left(-\tau^{\alpha}\right)^{n}}{n !} D_{t}^{n \alpha}\right) \mathbf{J} d u \\
& =\left(\sum_{n=0}^{+\infty} \frac{\left(-\tau^{\alpha}\right)^{n}}{n ! \Gamma(\beta)}\right)\left[\int_{0}^{+\infty} u^{\beta-1+n} d u\right]\left[D_{t}^{n \alpha} \mathbf{J}\right] \\
& =\left(\sum_{n=0}^{+\infty} \frac{\left(-\tau^{\alpha}\right)^{n} \Gamma(n+\beta)}{n ! \Gamma(\beta)}\right)\left[D_{t}^{n \alpha} \mathbf{J}\right]
\end{aligned}
$$

Eq. (20) can be reformulated by inducing a positive number $N_{\alpha, \beta}$ to truncate the summation series as ${ }^{5}$

$$
D_{t}^{\alpha, \beta} \mathbf{J}=\sum_{n=0}^{N_{\alpha, \beta}}\left(-\tau^{\alpha}\right)^{n}\left(\begin{array}{c}
n+\beta-1 \\
n
\end{array}\right)\left[D_{t}^{n \alpha} \mathbf{J}\right]
$$

To determine the accurate representation of the fractional differential operator, the optimal value of the summation number $N_{\alpha, \beta}$ is defined by the function of $\alpha$ and $\beta$ which satisfies the following error inequality ${ }^{5}$

$$
e r r=\sqrt{\frac{\int_{0}^{\omega_{\max }}\left|F(i \omega)-F^{\alpha}(i \omega)\right|^{2} d \omega}{\int_{0}^{\omega_{\max }}|F(i \omega)|^{2} d \omega}} \leq \delta
$$

in which $\delta$ is the positive cut-off value. The truncated binomial series are represented by ${ }^{5}$

$$
\begin{gathered}
F(i \omega)=\left[1+(i \omega \tau)^{\alpha}\right]^{-\beta} \\
F^{\alpha}(i \omega)=\sum_{n=0}^{N_{\alpha, \beta}}(-1)^{n}\left(\begin{array}{c}
n+\beta-1 \\
n
\end{array}\right)(i \omega \tau)^{n \alpha}
\end{gathered}
$$

The $\alpha$-order fractional differential operator $D_{t}^{n \alpha}$ in Eq. (21) can be defined by using Caputo's derivative as ${ }^{31}$

$$
D_{t}^{n \alpha} \mathbf{J}=\frac{1}{\Gamma(v-n \alpha)} \int_{0}^{t} \frac{\mathbf{J}(\delta)}{(t-\delta)^{n \alpha+1-v}} d \delta
$$

in which $v=\lceil n \alpha\rceil$ is the smallest integer that satisfies $v>n \alpha$. 
191

192

193

194

195

196

197

198

199

200

201

202

203

204

205

206

207

208

209

210

211

212

213

214

Based on the Grunwald-Letnikov operator, the derivatives in time domain can be represented by the limitation of the finite differences. For $\mathbf{J} \in C^{m}([0, T])$ and $v=\lceil n \alpha\rceil$, it is possible to extend the above equation as ${ }^{13,31}$

$$
D_{t}^{n \alpha} \mathbf{J}(t)=\lim _{m \rightarrow \infty} \frac{1}{h^{n \alpha}} \sum_{k=0}^{m-1} w_{k}^{(n \alpha)} \cdot \mathbf{J}(t-k \Delta t)
$$

in which $\Delta t=\frac{t}{m}$ represents the time interval and the $w_{k}^{(n \alpha)}$ represents the weight function. For the certain time instant $t=m \Delta t$, the Caputo's derivative can be reformulated by the discretized form as

$$
D_{t}^{n \alpha} \mathbf{J}\left(t_{m}\right)=(\Delta t)^{-n \alpha} \sum_{k=0}^{m-1} w_{k}^{(n \alpha)} \cdot \mathbf{J}\left(t_{m-k}\right)
$$

The weights $w_{k}^{(n \alpha)}$ in Eq. (26) can be equivalently defined by Gamma function ${ }^{31}$

$$
w_{k}^{(n \alpha)}=\left(\begin{array}{c}
k-n \alpha-1 \\
k
\end{array}\right)=\frac{\Gamma(k-n \alpha)}{\Gamma(-n \alpha) \Gamma(k+1)}
$$

and it can be evaluated in a recursive way as

$$
w_{0}^{(n \alpha)}=1, w_{k}^{(n \alpha)}=\left(1-\frac{n \alpha+1}{k}\right) w_{k-1}^{(n \alpha)}
$$

Therefore, the fractional differential expression in the time domain can be written as:

$$
D_{t}^{\alpha, \beta} \mathbf{J}=\sum_{n=0}^{N_{\alpha, \beta}}\left(-\tau^{\alpha}\right)^{n}\left(\begin{array}{c}
n+\beta-1 \\
n
\end{array}\right)(\Delta t)^{-n \alpha} \sum_{k=0}^{m-1} w_{k}^{(n \alpha)} \cdot \mathbf{J}\left(t_{m-k}\right)
$$

\section{FDTD formulation for wave propagation in $\mathrm{H}-\mathrm{N}$ media}

The time discretization form of particle velocity gradient $\mathbf{J}\left(t_{m-k}\right)=\frac{\partial \mathbf{v}\left(t_{m-k}\right)}{\partial x_{i}}$ can be expressed for a two-dimensional sound field as:

$$
\begin{gathered}
\frac{\partial u_{S}\left(t_{m-k}\right)}{\partial x} \approx \frac{\left.u_{S}\right|_{i+1 / 2, j} ^{m-k}-\left.u_{S}\right|_{i-1 / 2, j} ^{m-k}}{\Delta x} \\
\frac{\partial v_{S}\left(t_{m-k}\right)}{\partial y} \approx \frac{\left.v_{S}\right|_{i, j+1 / 2} ^{m-k}-\left.v_{S}\right|_{i, j-1 / 2} ^{m-k}}{\Delta y}
\end{gathered}
$$

The FDTD formulations of the sound pressure field and the particle velocity field in different temperature can be expressed by using Eqs. (3b), (3c), (15) and (29) by using Yee's algorithm as ${ }^{32}$

$$
\begin{aligned}
& \left.u_{S}\right|_{i+1 / 2, j} ^{m}=\left.u_{S}\right|_{i+1 / 2, j} ^{m-1}+\frac{\Delta t}{\rho_{s} \Delta x}\left(\left.p_{S}(T)\right|_{i+1, j} ^{m-1 / 2}-\left.p_{S}(T)\right|_{i, j} ^{m-1 / 2}\right) \\
& \left.v_{S}\right|_{i, j+1 / 2} ^{m}=\left.v_{S}\right|_{i, j+1 / 2} ^{m-1}+\frac{\Delta t}{\rho_{S} \Delta y}\left(\left.p_{S}(T)\right|_{i, j+1} ^{m-1 / 2}-\left.p_{S}(T)\right|_{i, j} ^{m-1 / 2}\right)
\end{aligned}
$$




$$
\begin{aligned}
\left.p_{S}(T)\right|_{i, j} ^{m+1 / 2} & =\left.p_{S}(T)\right|_{i, j} ^{m-1 / 2}+\frac{\Delta t}{\Delta x}\left(\frac{(3-v) E_{\infty}}{2(1-v)(1+v)}\right)\left(\left.u_{S}\right|_{i+1 / 2, j} ^{m}-\left.u_{S}\right|_{i-1 / 2, j} ^{m}\right)+\frac{\Delta t}{\Delta y}\left(\frac{E_{\infty}}{2(1-v)}\right)\left(\left.v_{S}\right|_{i, j+1 / 2} ^{m}-\left.v_{S}\right|_{i, j-1 / 2} ^{m}\right) \\
& +\frac{\Delta t}{\Delta x}\left(\frac{(3-v)\left(E_{0}-E_{\infty}\right)}{2(1-v)(1+v)}\right)\left[\sum_{n=0}^{N_{\alpha, \beta}}\left(-\tau(T)^{\alpha}\right)^{n}\left(\begin{array}{c}
n+\beta-1 \\
n
\end{array}\right)(\Delta t)^{-n \alpha}\left[\sum_{k=0}^{m-1} w_{k}^{(n \alpha)}\left(\left.u_{S}\right|_{i+1 / 2, j} ^{m-k}-\left.u_{S}\right|_{i-1 / 2, j} ^{m-k}\right)\right]\right] \\
& +\frac{\Delta t}{\Delta y}\left(\frac{E_{0}-E_{\infty}}{2(1-v)}\right)\left[\sum_{n=0}^{N_{\alpha, \beta}}\left(-\tau(T)^{\alpha}\right)^{n}\left(\begin{array}{c}
n+\beta-1 \\
n
\end{array}\right)(\Delta t)^{-n \alpha}\left[\sum_{k=0}^{m-1} w_{k}^{(n \alpha)}\left(\left.v_{S}\right|_{i, j+1 / 2} ^{m-k}-\left.v_{S}\right|_{i, j-1 / 2} ^{m-k}\right)\right]\right]
\end{aligned}
$$

217 The distribution of discretization points for sound pressure $p$, particle velocity $u$ and $v$ in 218 computational time and spatial domains are shown in Fig. 1

Fig. 1 Distribution of discretization points for sound pressure and particle velocity. (color online)

\section{Approximation of the FDTD method for wave propagation equation in fluid flow}

\section{2}

223

\section{A. Constitutive Equation of acoustic wave in fluid field}

In viscous fluid flow, the acoustic wave governing equations are constructed by the law of continuity, momentum and state. The Naiver-Stokes form for the governing equations for homogenous static fluid inside the rigid boundary condition can be expressed as ${ }^{3}$

$$
\begin{gathered}
\frac{\partial \mathbf{v}}{\partial t}=-\frac{1}{\rho_{F}} \nabla p_{F}+\eta \nabla^{2} \mathbf{v}+f \\
\frac{\partial \rho_{F}}{\partial t}=-\rho_{F} \nabla \mathbf{v} \\
\frac{\partial p_{F}}{\partial t}=c_{F}^{2} \frac{\partial \rho_{F}}{\partial t}
\end{gathered}
$$

in which $\mathbf{v}$ represents the particle velocity vector in the fluid flow, $\eta$ represents the viscosity resistance for homogenous fluid, $p_{F}$ represents the sound pressure in fluid region, and $\rho_{F}$ represents the fluid mass density. 
Neglecting the body force in the Eq. (32a) and minor density change of particles in Eq. (31b) for viscous fluid, the Navier-Stokes governing equations can be simplified as

$$
\begin{gathered}
\frac{\partial \mathbf{v}}{\partial t}=-\frac{1}{\rho_{F}} \nabla p_{F}+\eta \nabla^{2} \mathbf{v} \\
\frac{\partial p_{F}}{\partial t}=-K_{F} \nabla \mathbf{v}
\end{gathered}
$$

in which $K_{F}=\rho_{F} c_{F}^{2}$ represents the adiabatic bulk modulus of the fluid. For different thermal conditions, the relation between the sound velocity and the temperature of the distilled water can be expressed by ${ }^{33}$

$$
c_{F}(T)=1404+4.7 T-0.04 T^{2}
$$

in which $T$ represents the temperature in the range of $0^{\circ} \mathrm{C}<T<40^{\circ} \mathrm{C}$. The dependence of adiabatic bulk modulus on temperature then can be expressed as:

$$
K_{F}(T)=\rho_{F} c_{F}^{2}(T)
$$

For two-dimensional sound field, the acoustic wave governing equation in Cartesian coordination can be expanded as:

$$
\begin{gathered}
\frac{\partial p_{F}(T)}{\partial t}=-K_{F}(T)\left(\frac{\partial u_{F}}{\partial x}+\frac{\partial v_{F}}{\partial y}\right) \\
\frac{\partial u_{F}}{\partial t}=-\frac{1}{\rho_{F}} \frac{\partial p_{F}(T)}{\partial x}+\eta\left(\frac{\partial^{2} u_{F}}{\partial x^{2}}+\frac{\partial^{2} v_{F}}{\partial y^{2}}\right) \\
\frac{\partial v_{F}}{\partial t}=-\frac{1}{\rho_{F}} \frac{\partial p_{F}(T)}{\partial y}+\eta\left(\frac{\partial^{2} u_{F}}{\partial x^{2}}+\frac{\partial^{2} v_{F}}{\partial y^{2}}\right)
\end{gathered}
$$

in which $u_{F}$ represents the particle velocity in the x-direction and $v_{F}$ represents the particle velocity in the y-direction.

\section{B. FDTD formulation for wave propagation in fluid field}

The discretized points for sound pressure $p$ and particle velocity components $u$ and $v$ of two-dimensional fluid field in computational time and spatial domain are shown in Fig. 1. The second-order derivative for particle velocity components can be evaluated by using spatially central difference approximation as:

$$
\begin{aligned}
& \frac{\partial^{2} u_{F}(x, t)}{\partial x^{2}} \approx \frac{u_{F}(x+\Delta x, t)-2 u_{F}(x, t)+u_{F}(x-\Delta x, t)}{(\Delta x)^{2}} \\
& \frac{\partial^{2} v_{F}(y, t)}{\partial y^{2}} \approx \frac{v_{F}(y+\Delta y, t)-2 v_{F}(y, t)+v_{F}(y-\Delta y, t)}{(\Delta y)^{2}}
\end{aligned}
$$

Based on the point distribution in time and spatial, the two-dimensional FDTD formulation for viscous fluid flow can be subsequently obtained as:

$$
\left.u_{F}\right|_{i+1 / 2, j} ^{m}=\left.u_{F}\right|_{i+1 / 2, j} ^{m-1}-\frac{\Delta t}{\rho_{F} \Delta x}\left(\left.p_{F}(T)\right|_{i+1, j} ^{m-1 / 2}-\left.p_{F}(T)\right|_{i, j} ^{m-1 / 2}\right)+\eta \frac{\Delta t}{(\Delta x)^{2}}\left[\left(\left.u_{F}\right|_{i+3 / 2, j} ^{m-1}-\left.2 u_{F}\right|_{i+1 / 2, j} ^{m-1}+\left.u_{F}\right|_{i-1 / 2, j} ^{m-1}\right)\right]
$$

$$
+\eta \frac{\Delta t}{(\Delta y)^{2}}\left[\left(\left.v_{F}\right|_{i, j+3 / 2} ^{m-1}-\left.2 v_{F}\right|_{i, j+1 / 2} ^{m-1}+\left.v_{F}\right|_{i, j-1 / 2} ^{m-1}\right)\right]
$$




$$
\left.v_{F}\right|_{i, j+1 / 2} ^{m}=\left.v_{F}\right|_{i, j+1 / 2} ^{m-1}-\frac{\Delta t}{\rho_{F} \Delta x}\left(\left.p_{F}(T)\right|_{i, j+1} ^{m-1 / 2}-\left.p_{F}(T)\right|_{i, j} ^{m-1 / 2}\right)+\eta \frac{\Delta t}{(\Delta x)^{2}}\left[\left(\left.u_{F}\right|_{i+3 / 2, j} ^{m-1}-\left.2 u_{F}\right|_{i+1 / 2, j} ^{m-1}+\left.u_{F}\right|_{i-1 / 2, j} ^{m-1}\right)\right]
$$

$$
+\eta \frac{\Delta t}{(\Delta y)^{2}}\left[\left(\left.v_{F}\right|_{i, j+3 / 2} ^{m-1}-\left.2 v_{F}\right|_{i, j+1 / 2} ^{m-1}+\left.v_{F}\right|_{i, j-1 / 2} ^{m-1}\right)\right]
$$

$$
\left.p_{F}(T)\right|_{i, j} ^{m+1 / 2}=\left.p_{F}(T)\right|_{i, j} ^{m-1 / 2}-\frac{K_{F}(T) \Delta t}{\Delta x}\left(\left.u_{F}\right|_{i+1 / 2, j} ^{m}-\left.u_{F}\right|_{i-1 / 2, j} ^{m}\right)-\frac{K_{F}(T) \Delta t}{\Delta y}\left(\left.v_{F}\right|_{i, j+1 / 2} ^{m}-\left.v_{F}\right|_{i, j-1 / 2} ^{m}\right)
$$

262

263

264

265

266

267

268

269

270

271

272

273

274

\section{Continuity at interface between fluid and $\mathrm{H}-\mathrm{N}$ media}

By using the displacement equation in both fluid and solid domains, the continuity of particle velocity is enforced throughout. In the interface of the fluid and the solid, the normal component of the particle velocity should be continuous ${ }^{34}$. Therefore, for heterogeneous fluid-solid media, Eq. (31) can be used in solid H-N medium and Eq. (38) in fluid domain, along with the continuity boundary condition at the interface between two media. The kinematic continuity boundary condition can be imposed at the fluid-elastomer interface as ${ }^{35}$ :

$$
\underbrace{\mathbf{n}_{F} \frac{1}{\rho_{F}} \frac{\partial}{\partial t}\left(\frac{\partial p_{F}}{\partial x}\right)}_{\text {Fluid }}=\underbrace{-\mathbf{n}_{F} \frac{\partial^{2} u_{S}}{\partial t^{2}}}_{\text {Solid }}
$$

where $\mathbf{n}_{F}$ is an outward directed unit vector normal to the fluid boundaries. The traction continuity boundary condition can be achieved by imposing the following boundary condition at the solid-fluid interface as ${ }^{35}$

$$
\underbrace{n_{S_{1}} C_{1111} \frac{\partial u_{S}}{\partial x}+n_{S_{2}} C_{1122} \frac{\partial v_{S}}{\partial y}}_{\text {Solid }}=\underbrace{-n_{S_{1}} p_{F}}_{\text {Fluid }}
$$

where $\mathbf{n}_{S}$ is an outward directed unit vector normal to the solid boundaries. At the fluidelastomer interface it should be noted that $\mathbf{n}_{S}=-\mathbf{n}_{F}$.

Fig. 2 represents the normal particle velocity in the vicinity of the interface. For computational processing, the normal particle velocity passing through the interface can be calculated as ${ }^{15,36}$

$$
\underbrace{\left.u\right|_{i+1 / 2, j} ^{m}}_{\text {interface }}=\underbrace{\left.u\right|_{i+1 / 2, j} ^{m-1}}_{\text {interface }}-\frac{\Delta t}{\bar{\rho} \Delta x}\left(\left.p_{S}\right|_{i+1, j} ^{m-1 / 2}-\left.p_{F}\right|_{i, j} ^{m-1 / 2}\right)
$$

in which $\bar{\rho}=\left(\rho_{S}+\rho_{F}\right) / 2$. 


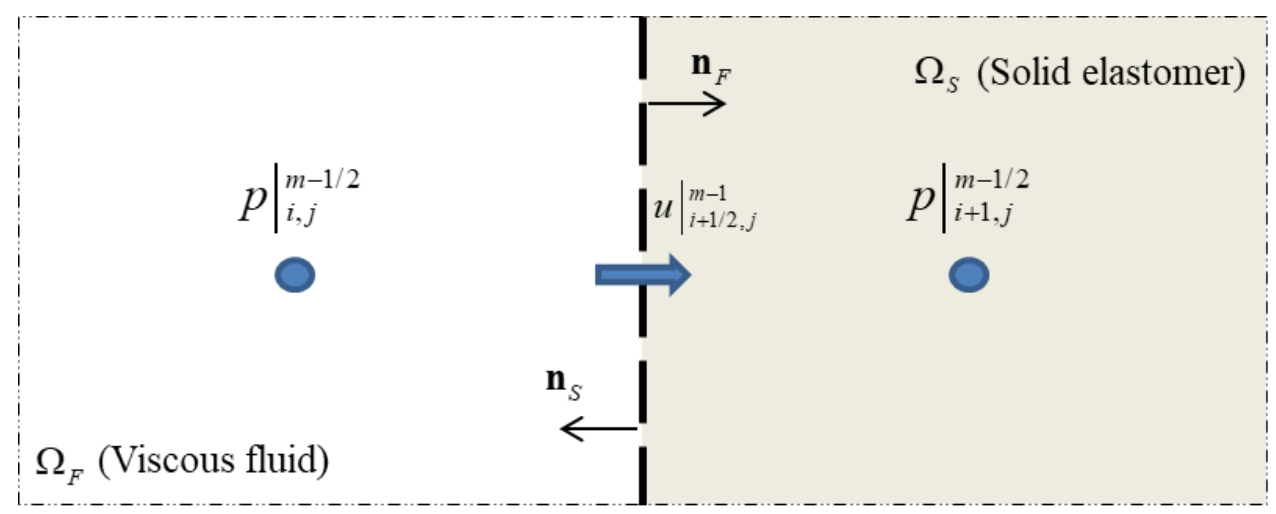

Fig. 2 The interface between the fluid field and solid elastomer. $\Omega_{F}$ represents the fluid domain and $\Omega_{S}$ represents the solid elastomer domain. $i$ represents the subscript of discrete point aligned with x-coordination. $j$ represents the subscript of discrete point aligned with y-coordination. (color online)

\section{Numerical algorithm}

The numerical algorithm for temperature-dependent FDTD formula is provided in following FDTD Algorithm. The spatial discretization for solid $\mathrm{H}-\mathrm{N}$ medium and fluid region is constructed prior to the time loops based on the geometry model, and remains the same during the time loops. During the numerical simulations, the particle velocities of two directions are stored in a time-dependent array. The summation of the particle velocities for each time step can be calculated from the array. In addition, the weight function $w_{k}^{(n \alpha)}$ will be updated for each time step by using the recursive relation for the Gamma function as shown in Eq. (28).

\section{Proposed FDTD Algorithm}

Input:

Geometry $L, W$ and material parameters $\alpha, \beta, E_{0}, E$

Spatial discretization $\Delta x, \Delta y$ and time interval $\Delta t$

Initial sound source condition

\section{Output:}

Sound pressure field, $p$ and particle velocity, $u, v$

1: for Each timestep $t \leq N_{t}, t++$ do

2: Calculate particle velocity gradient $\mathbf{J}\left(t_{m-k}\right)$ according to Eq. (30)

3: $\quad$ Update weight function $w_{k}^{(n \alpha)}$ according to Eq. (28)

4: $\quad$ Update $\alpha$-order fractional differential operator $D_{t}^{n \alpha}$ according to Eq. (26)

5: $\quad$ Update fractional differential operator $D_{t}^{\alpha, \beta} \mathbf{J}$ according to Eq. (29)

6: for Each spatial discrete point $k \leq N_{k}, k++$ do

7: $\quad$ Update pressure $\quad p$ according to Eq. (31a) and (36a)

8: $\quad$ Update pressure $u$ according to Eq. (31b) and (36a)

8: $\quad$ Update pressure $v$ according to Eq. (31c) and (36c)

9: $\quad$ end for 


\section{Numerical results}

In this section, numerical examples of $\mathrm{H}-\mathrm{N}$ dispersive media in a fluid region are presented by using the proposed temperature-dependent FDTD formulation. The mechanical and acoustical experiments are conducted by using two $\mathrm{H}-\mathrm{N}$ type specimens. Specimen A chloroprene rubber has diameter of $\mathrm{D}=114.8 \mathrm{~mm}$, thickness of $\mathrm{t}=48.6 \mathrm{~mm}$ and mass density of $\rho_{S}=1463.1 \mathrm{~kg} / \mathrm{m}^{3}$. Specimen B neoprene rubber has diameter of $\mathrm{D}=118.2 \mathrm{~mm}$, thickness of $\mathrm{t}=45.1 \mathrm{~mm}$ and mass density of $\rho_{S}=1457.9 \mathrm{~kg} / \mathrm{m}^{3}$.

\section{A. Dynamic Mechanical and Acoustic Performance Experiments}

The storage modulus $E^{\prime}$ and loss factor $\tan \delta$ are measured by using the Dynamic Mechanical Thermal Analysis (DMTA) experiment at various temperatures: $10{ }^{\circ} \mathrm{C}, 20{ }^{\circ} \mathrm{C}$, $30{ }^{\circ} \mathrm{C}, 40{ }^{\circ} \mathrm{C}$. The $\mathrm{H}-\mathrm{N}$ mechanical response for different temperatures can be established by using the environmental-independent $\mathrm{H}-\mathrm{N}$ parameters $\alpha, \beta, E_{0}, E_{\infty}$ and the environmentdependent parameter $\tau(T)$. These environmental-independent parameters can be optimized based on DMTA data including storage modulus and loss factor measured at $20{ }^{\circ} \mathrm{C}$ by using MATLAB optimization toolbox (shown in Appendix A). ${ }^{37}$ Table I summarizes the initial and optimized values of $\mathrm{H}-\mathrm{N}$ parameters.

Table I. Initial values and optimized values of model parameters

\begin{tabular}{cccccc}
\hline & Parameter Value & $E_{0}(\mathrm{~Pa})$ & $E_{\infty}(\mathrm{Pa})$ & $\alpha$ & $\beta$ \\
\hline $\begin{array}{c}\text { chloroprene } \\
\text { rubber }\end{array}$ & Initial & $3.275 \times 10^{7}$ & $3.106 \times 10^{9}$ & 0.600 & 0.510 \\
\hline neoprene & Optimized & $2.344 \times 10^{7}$ & $4.997 \times 10^{9}$ & 0.793 & 0.336 \\
rubber & Initial & $3.395 \times 10^{7}$ & $6.292 \times 10^{8}$ & 0.288 & 0.628 \\
& Optimized & $2.853 \times 10^{7}$ & $4.994 \times 10^{8}$ & 0.517 & 0.229 \\
\hline
\end{tabular}

The empirical constants $C_{1}^{\prime}$ and $C_{2}^{\prime}$ for the WLF shift function at reference temperature $20{ }^{\circ} \mathrm{C}$ are calculated by using Eq. (12) as $C_{1}^{\prime}=21.3, C_{2}^{\prime}=42.3$ for specimen A chloroprene rubber and $C_{1}^{\prime}=15.9, C_{2}^{\prime}=67.1$ for specimen $\mathrm{B}$ neoprene rubber. The transfer factor, $\phi_{T_{M}}$ based on the reference temperature, $20^{\circ} \mathrm{C}$ can be calculated by using Eq. (13). The relaxation time, $\tau(T)$ for different temperatures can be obtained by using Eq. (14).

Based on H-N mechanical response described in Section II.B, the storage modulus $E^{\prime}$ is calculated by using Eq. (15) and the loss factor $\tan \delta$ is calculated by using Eq. (9). Figs. 4-5 show the storage modulus $E^{\prime}$ and loss factors $\tan \delta$ for two H-N type rubbers at different temperatures. It is observed that the mechanical properties at different temperatures calculated by the optimized H-N parameters are well compared with the experimental data, validating the efficiency of the H-N parameters.

Furthermore, the acoustic absorption coefficients for both $\mathrm{H}-\mathrm{N}$ type media are also measured at various temperatures by using the water-filled impulse duct. The water-filled impulse duct is utilized with length of $3 \mathrm{~m}$ and the effective frequency ranges from $50 \mathrm{~Hz}-$ $12.7 \mathrm{kHz}$. The two-hydrophone impulse method is adopted in the measurements. 


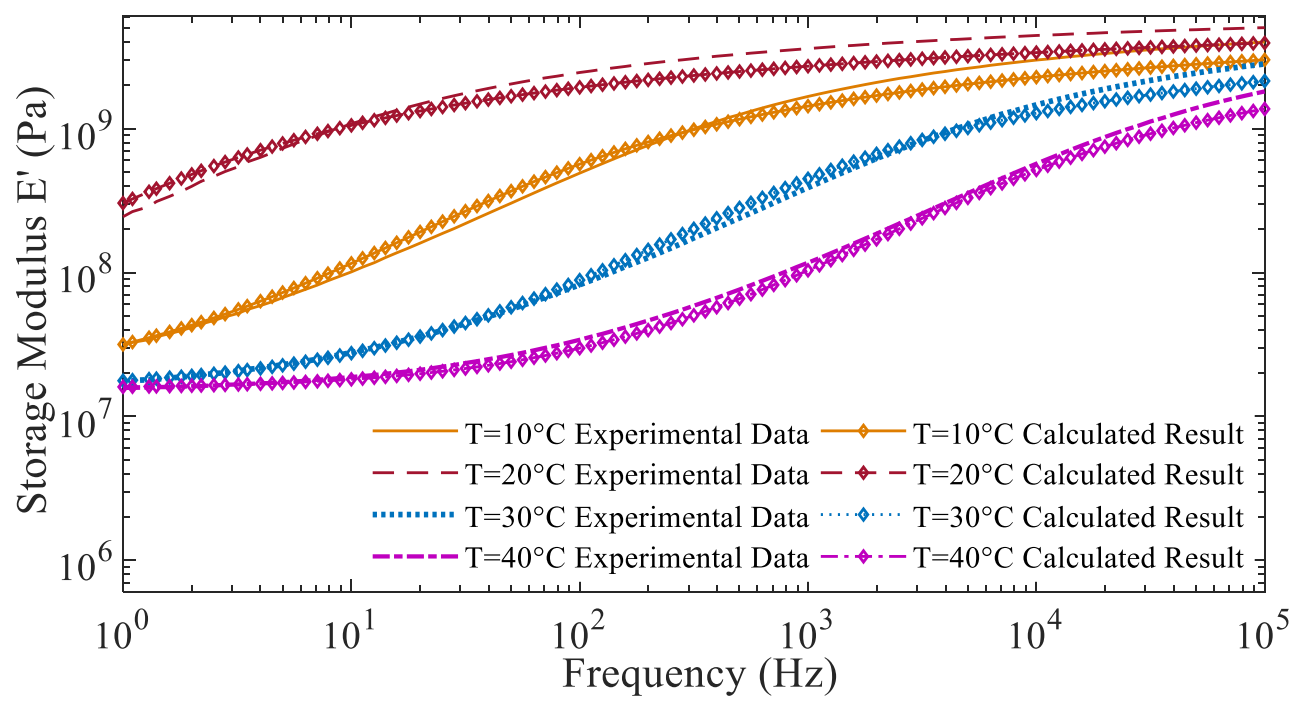

(a)

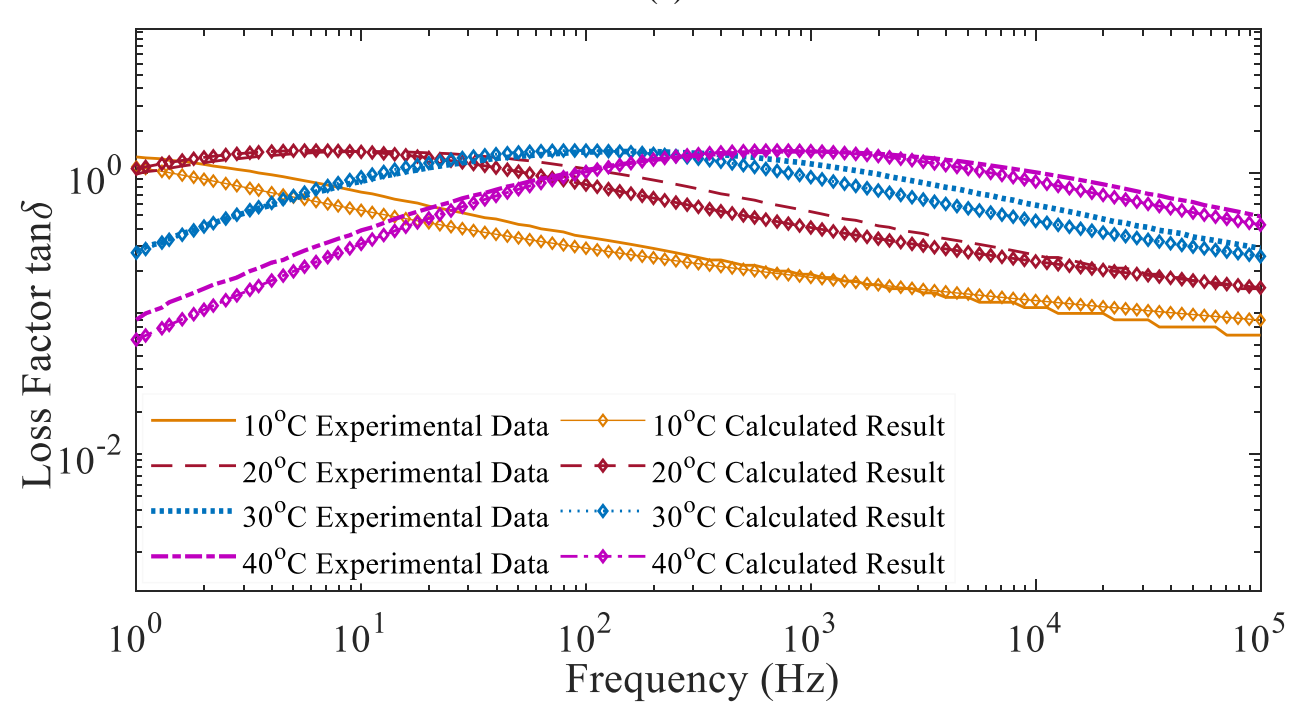

(b)

Fig. 4 Comparison of calculated (a) storage modulus and (b) loss factor of chloroprene rubber with experimental results at different temperatures. (color online)

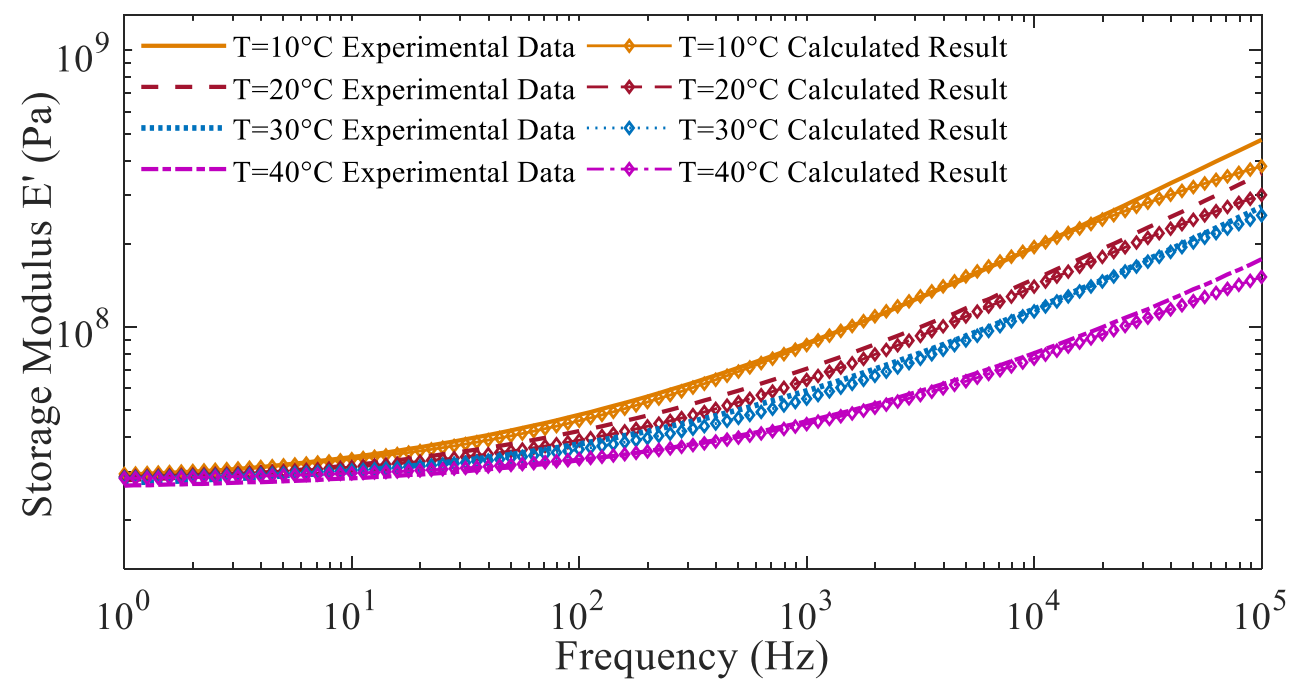




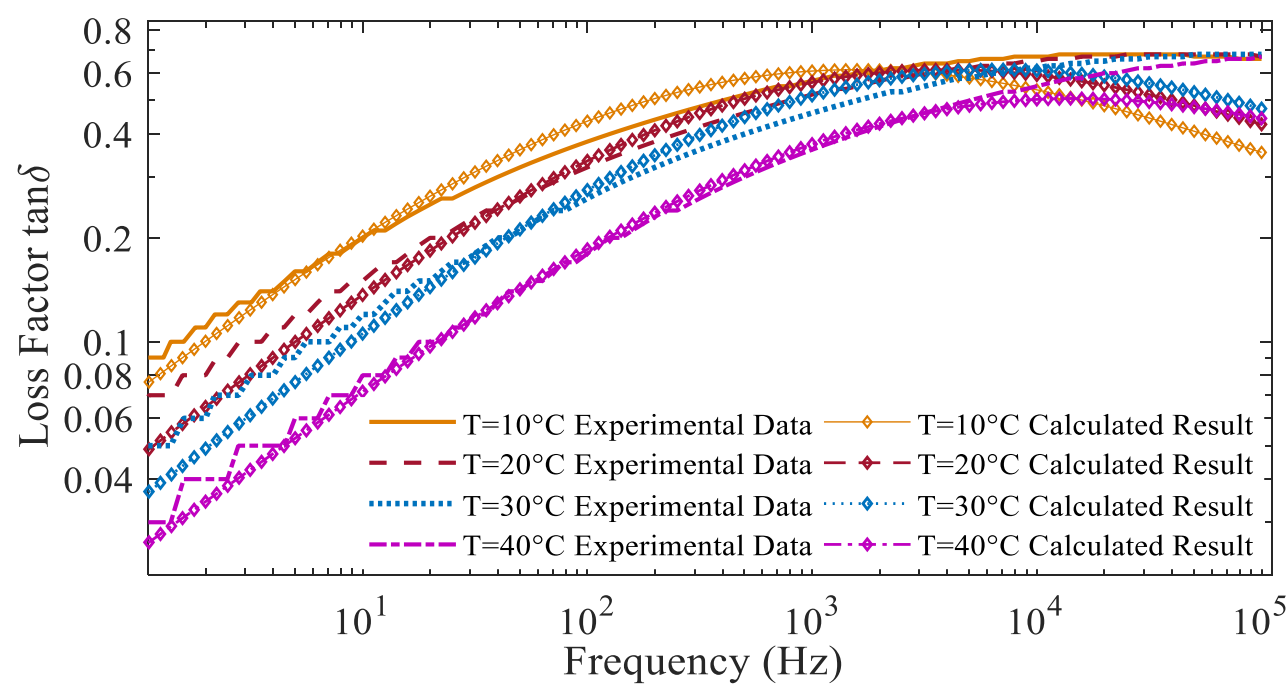

(b)

Fig. 5 Comparison of calculated (a) storage modulus and (b) loss factor of neoprene rubber with experimental results at different temperatures. (color online)

\section{B. Validation for Proposed FDTD scheme}

The proposed FDTD scheme is firstly assessed by considering the sound field in a narrow, fluid-filled waveguide, terminated on the right by an elastomer specimen as shown in Fig. 6(a). In the numerical simulations, the sound propagation medium consists of the fluid region and the solid elastomer. The acoustic coefficients of solid elastomer at different temperatures are calculated by using normal incident wave, and compared with the measured acoustic coefficient to validate the efficiency of proposed FDTD scheme. The length of the propagation path is specified as $L=3 \mathrm{~m}$ and the width is specified as the diameter $\mathrm{D}$ of the sample. The sample is placed in the right-hand termination of the simulated sound field. The thickness of the solid elastomer domain is identical with the thickness of sample in the experiment. The upper, bottom, left boundaries, along with the back surface of the elastomer are set to be the rigid walls. Sound source is implemented at the surface $S$ as an array which can be considered as a normal incident plane wave. The distance from the left boundary to the sound source is specified as $L_{\mathrm{S}}=9.0 \mathrm{~m}$. Point $\mathrm{R}$ represents the receiving point and the distance from $\mathrm{R}$ to the left boundary is specified as $L_{\mathrm{R}}=9.66 \mathrm{~m}$ for specimen $\mathrm{A}$ and $L_{\mathrm{R}}=9.81 \mathrm{~m}$ for specimen $\mathrm{B}$ in order to separate the incident and reflected wave. The absorption coefficient can be calculated by using the incident and reflected wave received at the point $\mathrm{R}$ and compared with the measurement data.

Furthermore, a broader 2-D simulation are also presented by using the proposed FDTD scheme for the water-filled acoustic duct terminated with two kinds of $\mathrm{H}-\mathrm{N}$ type media. The sound field at different temperature can be observed which is consistent with the actual water-filled standing wave duct applied in hydroacoustic measurements for $\mathrm{H}-\mathrm{N}$ media. The thermal effect on the velocity field is investigated by using the simulation results which can be related to the specific material property of the $\mathrm{H}-\mathrm{N}$ dispersive media. Besides, the relative error analysis is implemented based on the simulated sound pressure field to validate the accuracy of the proposed FDTD scheme. ${ }^{4,5}$ Fig. 6(b) shows the simulated space of this study 
cases. According to the size of the experimental water-filled standing wave duct, the length of the sound field is specified as $L=10 \mathrm{~m}$ and the width is specified as $W=0.12 \mathrm{~m}$. The thickness of the specimen is specified as $t=0.2 \mathrm{~m}$. The sound propagation medium is comprised of the fluid domain and the material domain. The other three boundaries and the back surface of the H-N media are set as rigid walls. The sound source is implemented at the surface $\mathrm{S}$ which is specified as the left boundary of the simulated field. The spatial pressure and the particle velocity distribution are simulated in these cases.

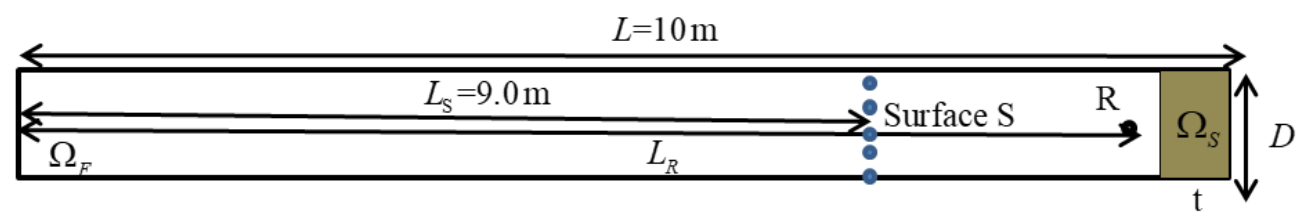

(a)

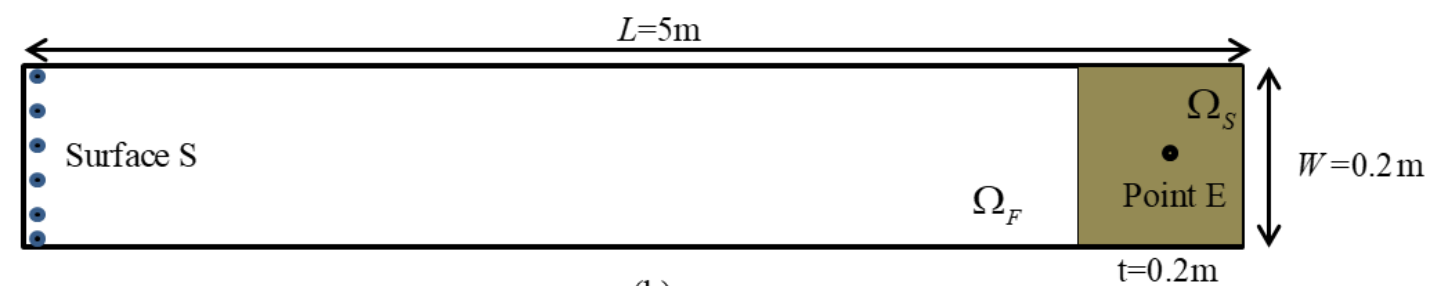

(b)

Fig. 6 Computational spatial of FDTD analysis for the (a) narrow spatial domain and (b) broader spatial domain. (color online)

In FDTD analysis, the discrete spatial interval is specified as $\Delta x=\Delta y=0.002 \mathrm{~m}$. The discrete time interval is specified as $\Delta t=4.8766 \times 10^{-7} \mathrm{~s}$ which satisfies the stability condition (shown in Appendix B)

$$
\begin{gathered}
\sqrt{\frac{C_{1111}+C_{1122}}{\rho_{s}}} \frac{\Delta t}{\Delta x} \leq 1 \\
\sqrt{\frac{K_{F}}{\rho_{F}} \frac{\Delta t}{\Delta x}} \leq \frac{1}{\sqrt{2}}
\end{gathered}
$$

A Gaussian pulse is utilized at each discrete point $\left(S_{x}, 1\right),\left(S_{x}, 2\right), \ldots,\left(S_{x}, A_{y}\right)$ as the sound source which can be expressed by

$$
p_{i, j}^{n+1 / 2}=10 \exp \left(-S\left(n \Delta t-\tau_{0}\right)^{2}\right)
$$

where $i$ equals to $S_{x}$ with $S_{x}$ representing the number of discrete grids up to the surface $S$ in x-direction. Here $S_{x}=L_{S} / \Delta x$ for the simulations shown in Fig. 6(a) and $S_{x}=\Delta x$ for the simulations shown in Fig. 6(b), respectively. $j$ is from 1 to $A_{y}$ with $A_{y}$ representing the total number of discrete grids in y-direction. Here $A_{y}=D / \Delta x$ for the simulation shown in Fig. 6(a) and $A_{y}=W / \Delta x$ for the simulation shown in Fig. 6(b), respectively. The constants for the pulse characteristic in frequency domain are chosen as $\tau_{0}=180 \Delta t$ and $S=\left(4 / \tau_{0}\right)^{2}$, and $n$ is specified as time steps in the FDTD simulation. 
In the simulation for fluid domain, the viscous fluid is specified as pure water with viscosity resistance $\eta=1.01 \times 10^{-6} \mathrm{~Pa} \cdot \mathrm{s}$ and the mass density $\rho_{F}=997 \mathrm{~kg} / \mathrm{m}^{3}$. The longitudinal wave velocity $c_{F}$ can be calculated by using Eq. (34) for different temperatures. In the simulations for $\mathrm{H}-\mathrm{N}$ type solid domain, the mass density of sample is utilized as $\rho_{S}$. The Poisson's ratio for both $\mathrm{H}-\mathrm{N}$ media is chosen as 0.33 . The $\mathrm{H}-\mathrm{N}$ parameters $\alpha, \beta, E_{0}, E_{\infty}, \tau$ for both H-N media used in Eq. (31a) are shown in Table I. The summation number in Eq. (31a) is calculated as $N_{\alpha, \beta}=7$ for chloroprene rubber and $N_{\alpha, \beta}=9$ for neoprene rubber.

\section{B.1 Specimen A chloroprene rubber}

Based on the proposed FDTD formulations shown in Eq. (31) and Eq. (38), the sound wave propagation in the fluid-filled waveguide terminated by specimen A chloroprene rubber are simulated. The sound pressure at the receiving point in different temperatures are obtained. The time-domain waveform is shown in Fig. 7. It indicates that the incident wave $(0.6 \mathrm{~ms}-$ $0.8 \mathrm{~ms})$ and the reflected wave $(1.0 \mathrm{~ms}-1.4 \mathrm{~ms})$ are completely separated, and the amplitude of the reflected wave increases with the temperature.

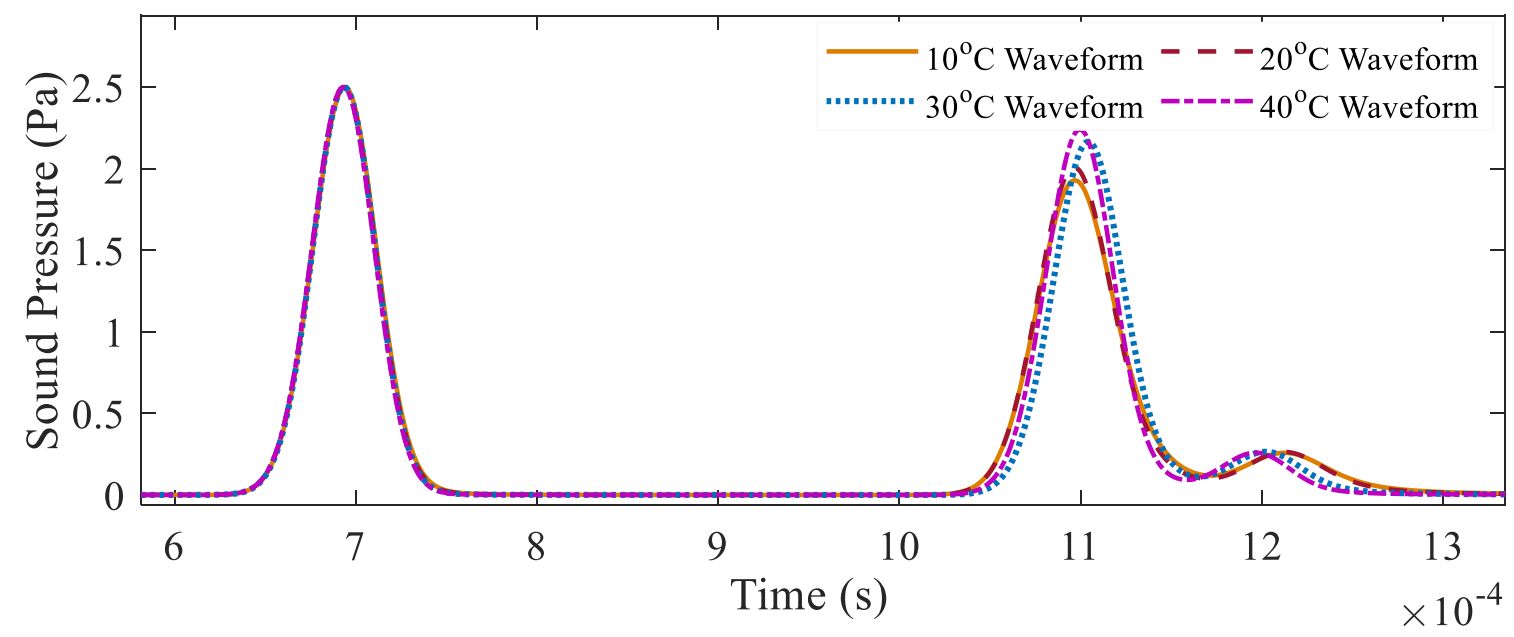

Fig. 7 The waveform of sound pressure at the receiving point for chloroprene rubber. (color online)

Ignoring the sound absorption caused by viscous water, the normal incident absorption coefficient $A(\omega)$ of the specimen in frequency domain then can be calculated as

$$
A(\omega)=1-\left|P_{\text {ref }}(\omega) / P_{\text {inc }}(\omega)\right|^{2}
$$

in which $P_{r e f}(\omega)$ and $P_{i n c}(\omega)$ represent the frequency response of the incident and reflected sound pressures respectively.

The calculated and measured absorption coefficients in different temperatures are presented in Fig. 8. As it can be seen from Fig. 8, the absorption coefficients for chloroprene rubber calculated by the proposed FDTD method are in good agreement with the measurement data at various temperatures. The calculated results represented by solid lines cover the whole frequency range from $1 \mathrm{~Hz}-10 \mathrm{kHz}$, while the experimental results represented by dash lines with stars are discrete data from $400 \mathrm{~Hz}$. The calculated results compare well with the experimental results with the deviation less than $8 \%$ for almost whole frequency range for each temperature condition. The maximum deviation can be observed 
near the absorption peak in the range of $2.9 \mathrm{kHz}-3.5 \mathrm{kHz}$. The absorption peak appears in almost the same frequency range at various temperatures, but the amplitude of the absorption coefficient decreases as the temperature increases. The comparisons of the simulated results and the experimental results validate the proposed FDTD formulation both for H-N type elastomer and viscous water.

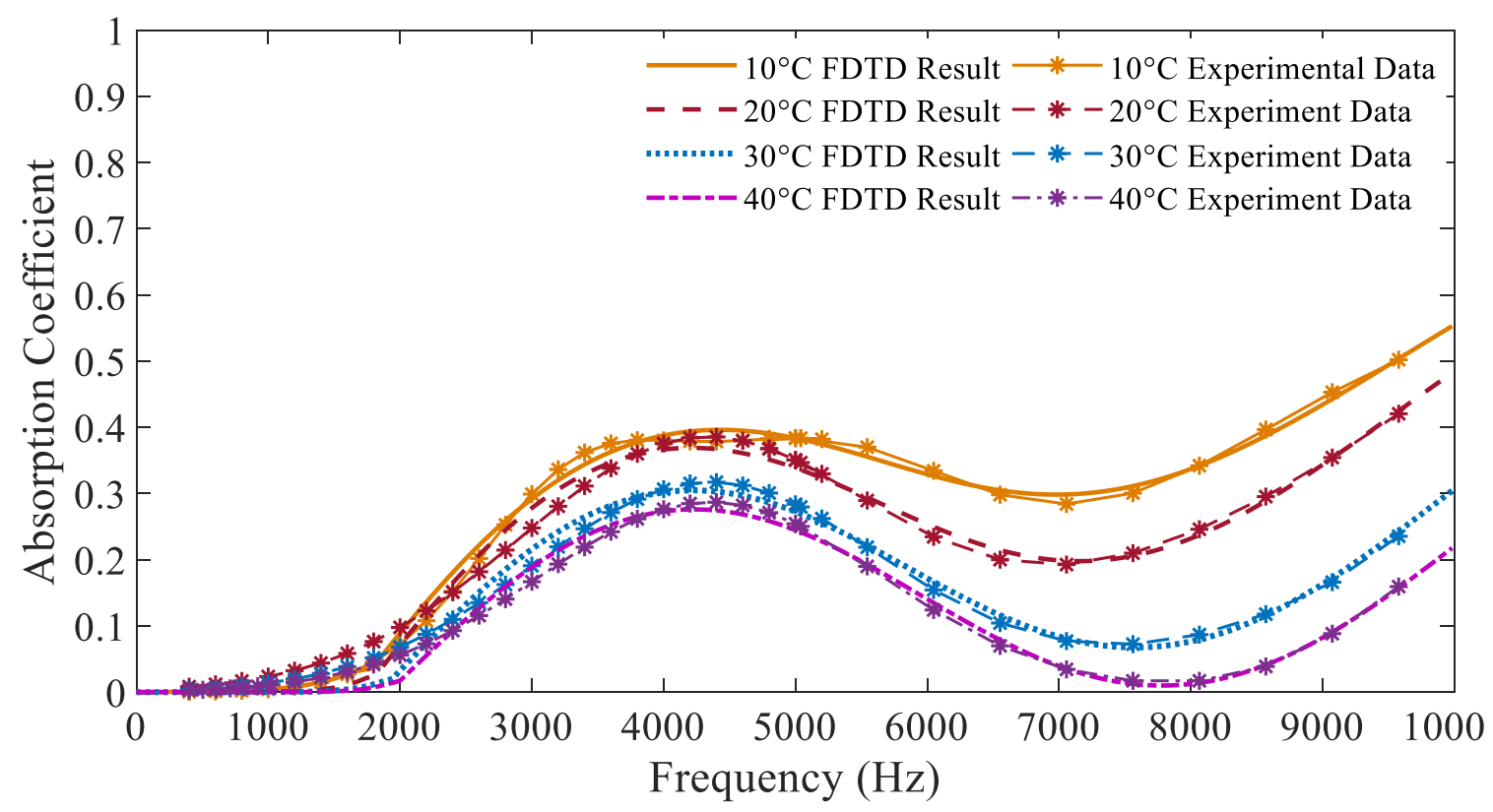

Fig. 8 Absorption coefficient for chloroprene rubber by FDTD method and experimental result. (color online)

For the broader 2-D sound field, the spatial pressure distribution near the $\mathrm{H}-\mathrm{N}$ specimen at $3.75 \mathrm{~ms}$ and $5.0 \mathrm{~ms}$ in $10{ }^{\circ} \mathrm{C}, 20^{\circ} \mathrm{C}, 30{ }^{\circ} \mathrm{C}$ and $40{ }^{\circ} \mathrm{C}$ are shown in Figs. $9-10$ respectively. Figs. 9-10 show that the proposed FDTD formulation can be used to simulate the sound field in the water-filled standing wave duct when applied in the hydroacoustic measurement for $\mathrm{H}$ $\mathrm{N}$ media, considering absorption and reflection at the boundary of the specimen. For each temperature condition, the sound wave starts to propagate into the $\mathrm{H}-\mathrm{N}$ specimen before 3.75 $\mathrm{ms}$ and then propagates back to the fluid domain before $5.0 \mathrm{~ms}$. By comparison of the spatial pressure distribution in different temperatures, it can be observed that the absolute sound pressure amplitude within $\mathrm{H}-\mathrm{N}$ media decreases in higher temperature, indicating that the sound energy propagating back into fluid flow increases with the temperature increases. This characteristic is identical to the variation of material properties in different temperatures.

The spatial distributions of particle velocity in two propagating directions at $2.5 \mathrm{~ms}$ in $40{ }^{\circ} \mathrm{C}$ are shown in Fig. 11. The spatial distribution patterns in different temperatures are similar. The mean values of particle velocity at different temperatures are summarized in Table II by using ${ }^{38}$

$$
u_{\text {average }}=\sum_{j=1}^{A_{y}} \sum_{i=1}^{A_{x}} u_{i, j} /\left(A_{x} A_{y}\right)
$$

in which $A_{x}$ representing the total number of discrete grids in x-direction and $A_{y}$ representing the total number of discrete grids in y-direction. The difference between each temperature condition mainly reflects the thermal effect on the $\mathrm{H}-\mathrm{N}$ dispersive media. As it 
450

457

can be seen from the Table II, the mean value of the horizontal particle velocity $u$ increases from $2.16 \times 10^{-5} \mathrm{~m} / \mathrm{s}$ to $2.41 \times 10^{-5} \mathrm{~m} / \mathrm{s}$, nearly $11.5 \%$ with the temperature increases from $10{ }^{\circ} \mathrm{C}$ to $40{ }^{\circ} \mathrm{C}$. On the contrary, the mean value of the vertical particle velocity $v$ almost remains the same. It indicates that higher temperature leads to more significant particle vibration aligned with the propagation direction, ${ }^{39}$ but has less effect on the particle velocity that perpendicular to the propagating direction.

Table II. Mean particle velocity for specimen A at $5.0 \mathrm{~ms}$ in different temperatures

\begin{tabular}{ccccc}
\hline $\begin{array}{c}\text { Specimen A chloroprene } \\
\text { rubber }\end{array}$ & $10{ }^{\circ} \mathrm{C}$ & $20{ }^{\circ} \mathrm{C}$ & $30{ }^{\circ} \mathrm{C}$ & $40{ }^{\circ} \mathrm{C}$ \\
\hline$u$ & $3.79 \times 10^{-5} \mathrm{~m} / \mathrm{s}$ & $3.86 \times 10^{-5} \mathrm{~m} / \mathrm{s}$ & $3.96 \times 10^{-5} \mathrm{~m} / \mathrm{s}$ & $4.07 \times 10^{-5} \mathrm{~m} / \mathrm{s}$ \\
\hline$v$ & $1.22 \times 10^{-8} \mathrm{~m} / \mathrm{s}$ & $1.22 \times 10^{-8} \mathrm{~m} / \mathrm{s}$ & $1.24 \times 10^{-8} \mathrm{~m} / \mathrm{s}$ & $1.26 \times 10^{-8} \mathrm{~m} / \mathrm{s}$ \\
\hline
\end{tabular}

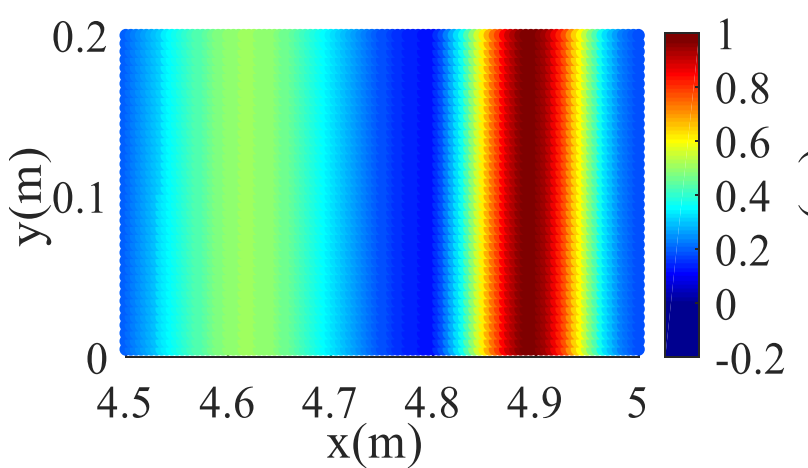

(a)

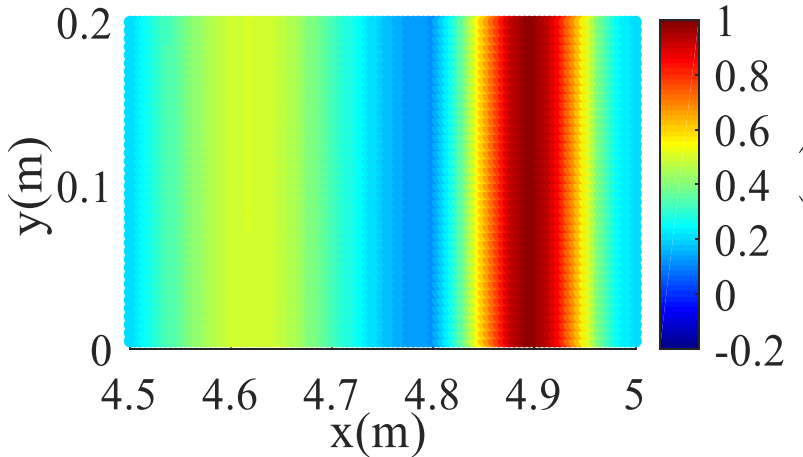

(c)

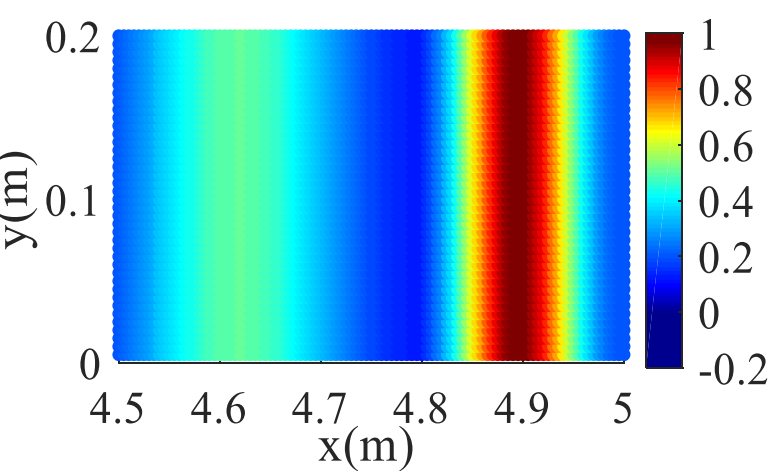

(b)

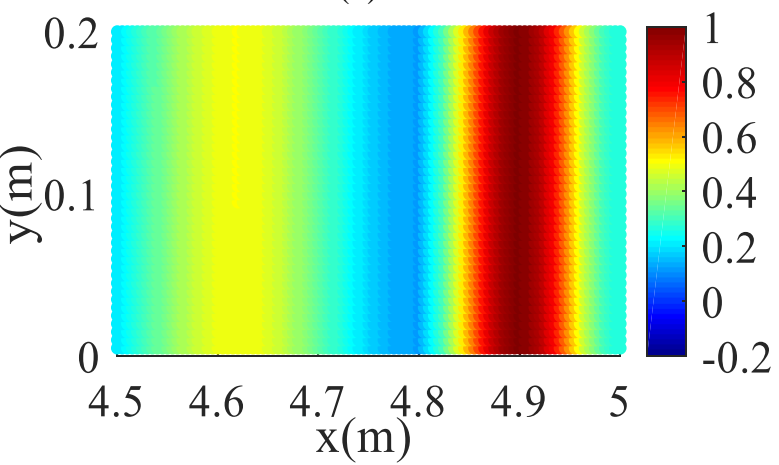

(d)

Fig. 9 The spatial distribution of sound pressure at $3.75 \mathrm{~ms}$ for (a) $10^{\circ} \mathrm{C}$ (b) $20^{\circ} \mathrm{C}$ (c) $30^{\circ} \mathrm{C}$ (d) $40{ }^{\circ} \mathrm{C}$ (color online)

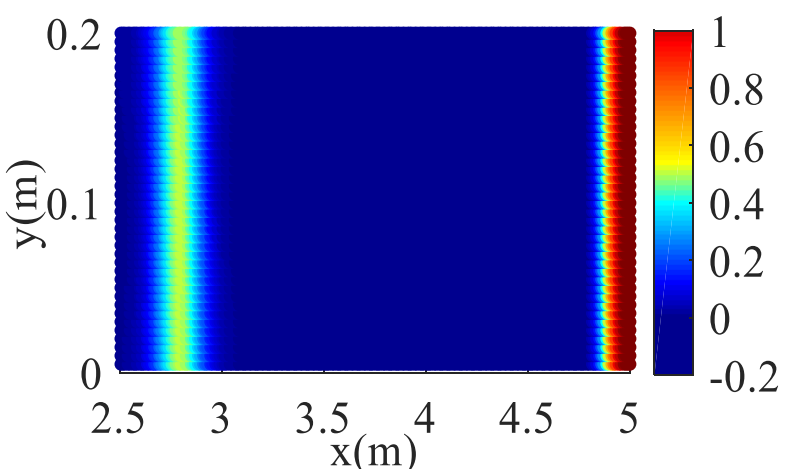

(a)

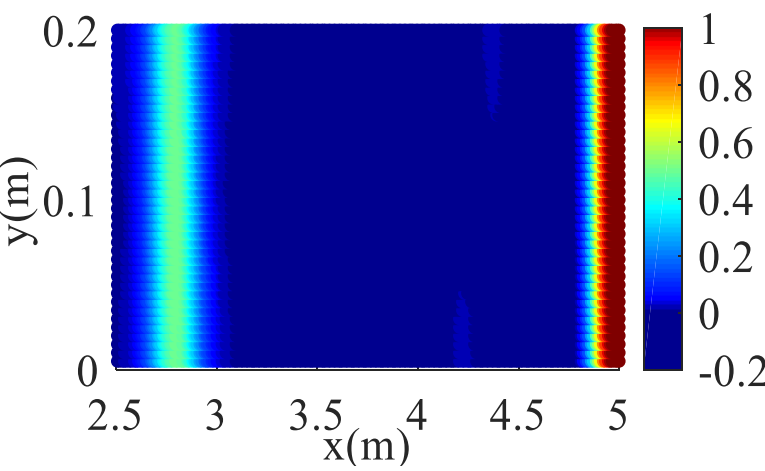

(b) 


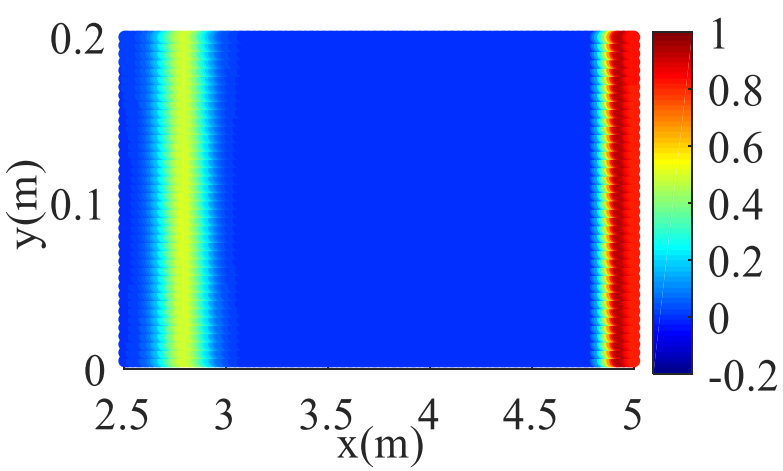

(c)

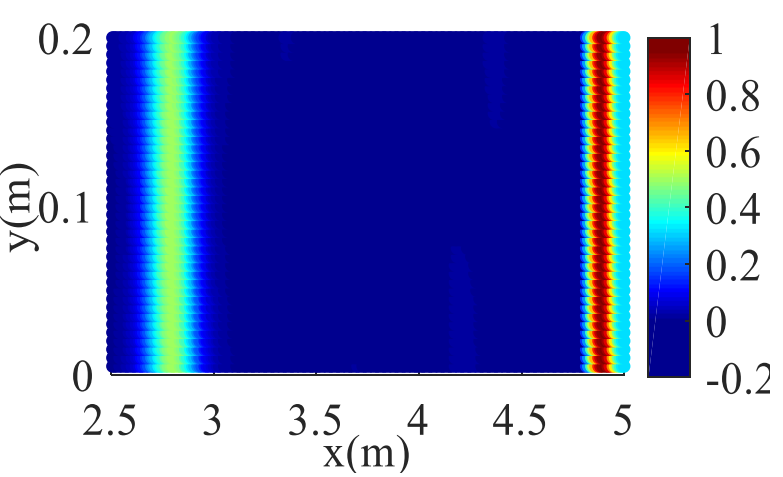

(d)

Fig. 10 The spatial distribution of sound pressure at $5.0 \mathrm{~ms}$ for (a) $10^{\circ} \mathrm{C}$ (b) $20^{\circ} \mathrm{C}$ (c) $30^{\circ} \mathrm{C}$ (d) $40{ }^{\circ} \mathrm{C}$ (color online)

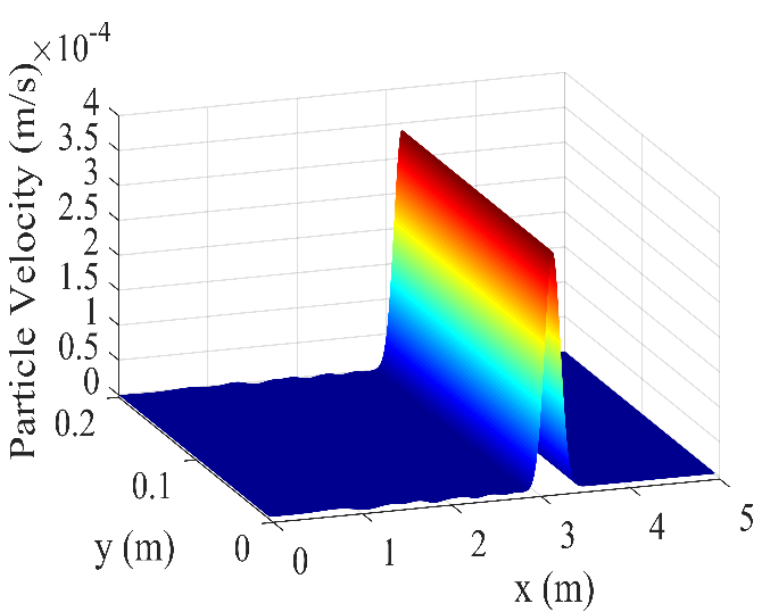

(a)

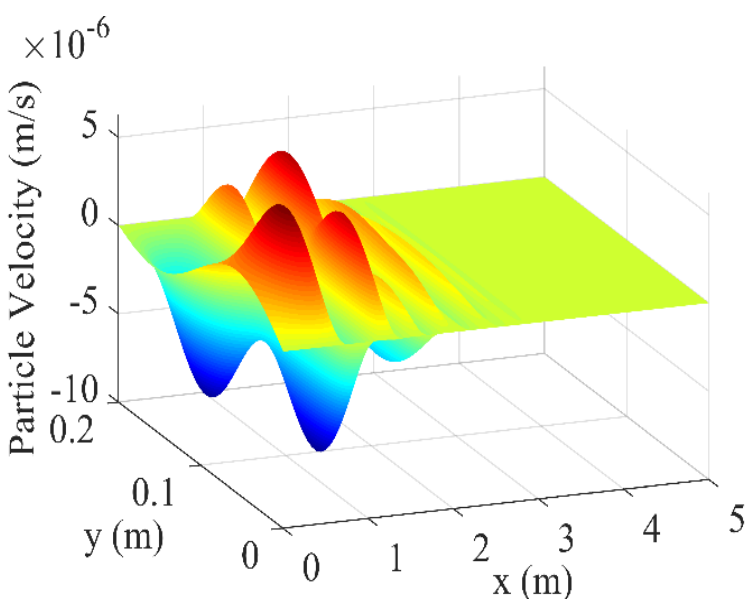

(b)

Fig. 11 The spatial distribution of particle velocities for (a) $u$ in the wave propagating direction and (b) $v$ perpendicular to the propagating direction. (color online)

\section{B.2 Specimen B neoprene rubber}

In this section the sound wave propagation in fluid field and neoprene rubber is simulated. Fig. 12 shows the sound pressure at the receiving point in different temperatures. As it can be seen from Fig. 12, the incident wave $(0.6 \mathrm{~ms}-0.8 \mathrm{~ms})$ and the reflected wave $(1.7 \mathrm{~ms}-2.2$ $\mathrm{ms})$ are completely separated. The normal incident absorption coefficient of the neoprene rubber in frequency domain is subsequently calculated. The calculated and measured absorption coefficients in different temperatures are presented in Fig. 13.

As it can be seen from Fig. 13, the absorption coefficients for neoprene rubber calculated by the proposed FDTD method compare well with the measurement data at various temperatures. The deviations are less than $3 \%$ in almost whole frequency range, and the maximum deviation can be observed at the absorption trough of the first mode for each temperature. The absorption peak appears in almost the same frequency range at various temperatures, and the amplitude of the absorption coefficient increases as the temperature increases. The comparisons of the simulated results and the experimental results highlight the efficiency of the proposed FDTD formulation. 


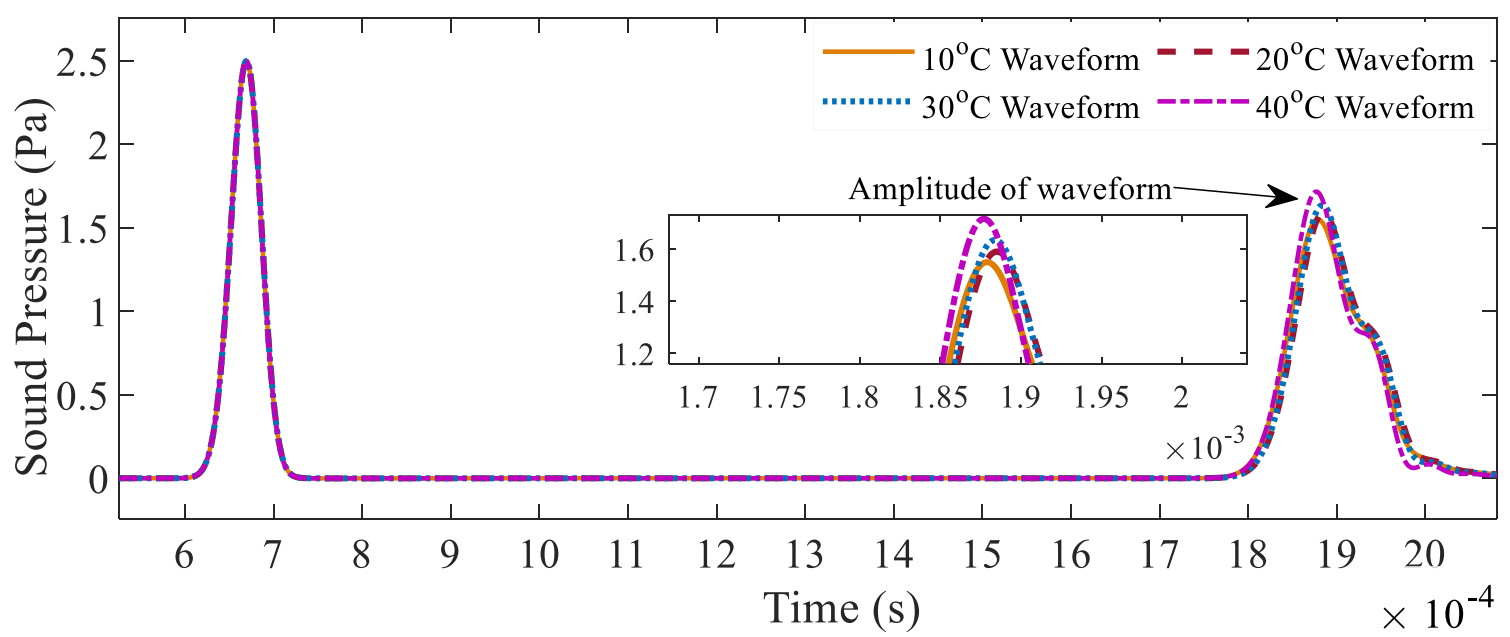

Fig. 12 The waveform of sound pressure at the receiving point for neoprene rubber. (color online)

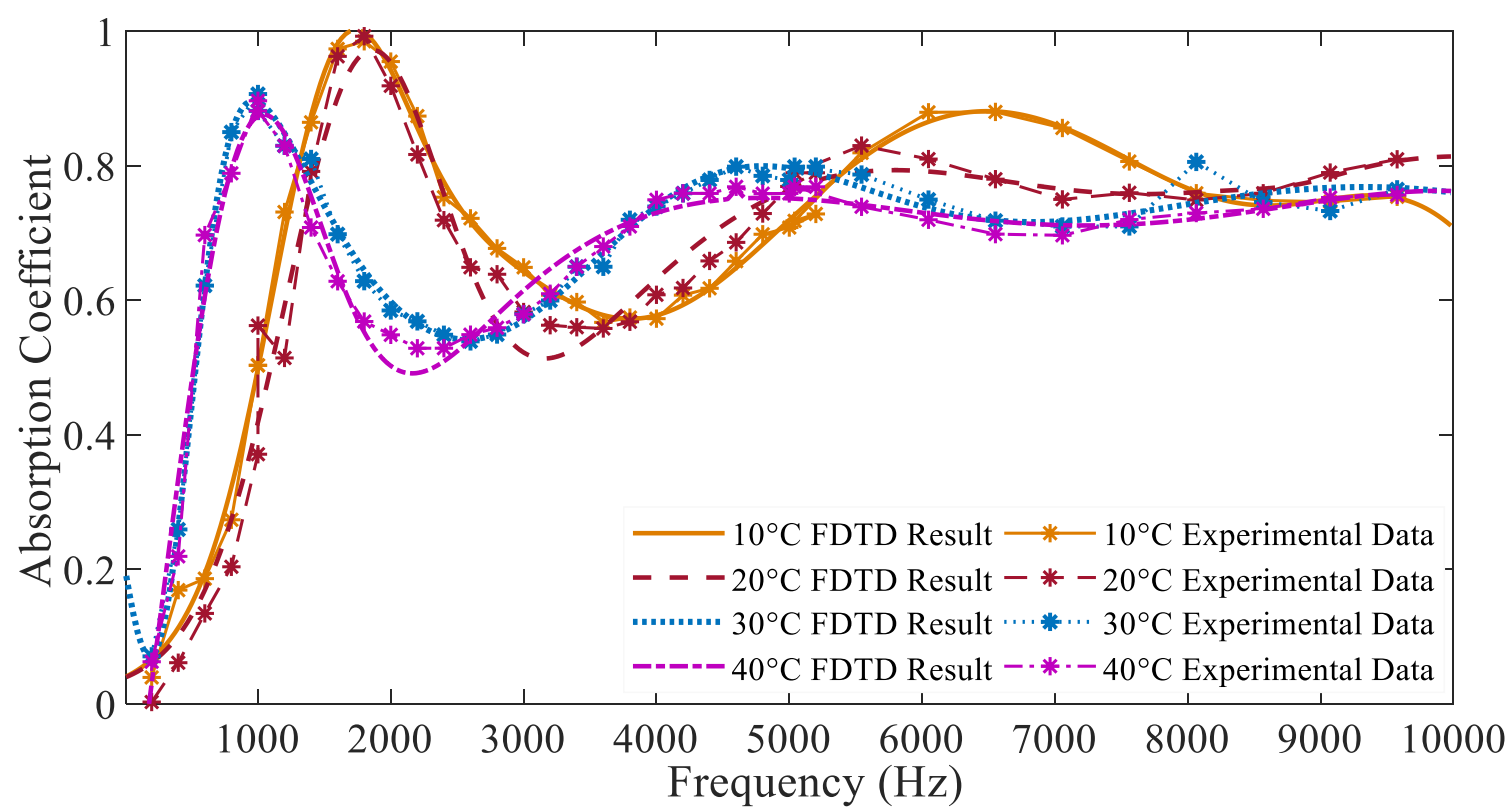

Fig. 13 Absorption coefficient for neoprene rubber by FDTD method and experimental result. (color online)

For the broader 2-D sound field, the spatial pressure distribution at $3.75 \mathrm{~ms}$ and $5.0 \mathrm{~ms}$ in $10^{\circ} \mathrm{C}, 20^{\circ} \mathrm{C}, 30^{\circ} \mathrm{C}$ and $40^{\circ} \mathrm{C}$ are shown in Figs. $14-15$ respectively. The spatial distributions of particle velocity in two propagating directions at $2.5 \mathrm{~ms}$ in $40{ }^{\circ} \mathrm{C}$ are also shown in Fig. 16 respectively. By comparing with Figs.9-10, the moderate fluctuation of sound pressure can be observed from Figs.14-15. It indicates that thermal conditions have noticeable effect on the wave propagation within the chloroprene rubber than that within the neoprene rubber. These results agree well with the greater relaxation time calculated for chloroprene rubber.

Table III shows the mean values of particle velocity at different temperatures. The mean value of the particle velocity $u$ increases from $3.79 \times 10^{-8} \mathrm{~m} / \mathrm{s}$ to $4.07 \times 10^{-8} \mathrm{~m} / \mathrm{s}$, nearly $7.3 \%$ with the temperature increases from $10{ }^{\circ} \mathrm{C}$ to $40{ }^{\circ} \mathrm{C}$. For vertical particle velocity $v$, the mean value of the vertical particle velocity $v$ almost remains the same. It also proves the higher temperature leads to strong particle vibration ${ }^{39}$ and the thermal effect shows significant influence on the particle velocity of chloroprene rubber than that of neoprene rubber. 
508

509

510

511

512

513

514

515

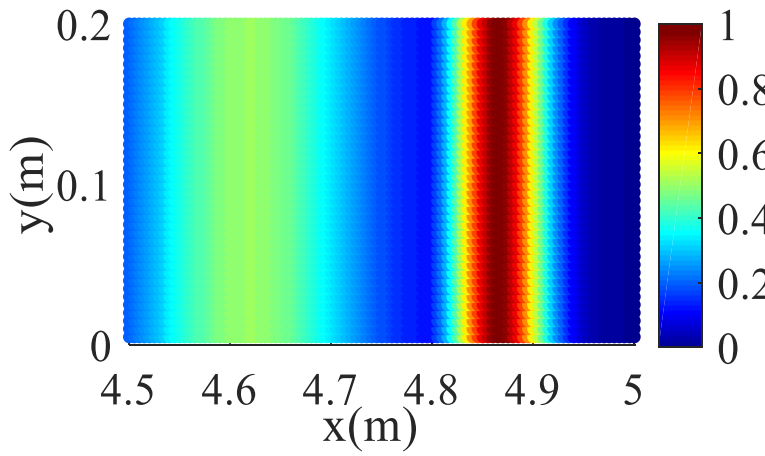

(a)

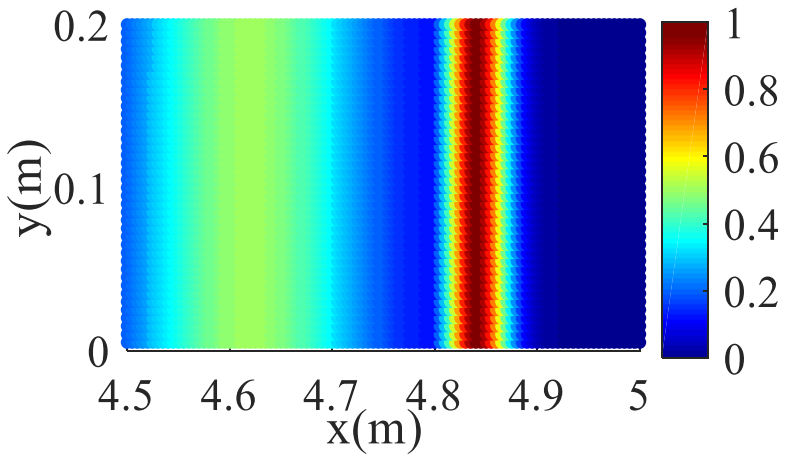

(c)

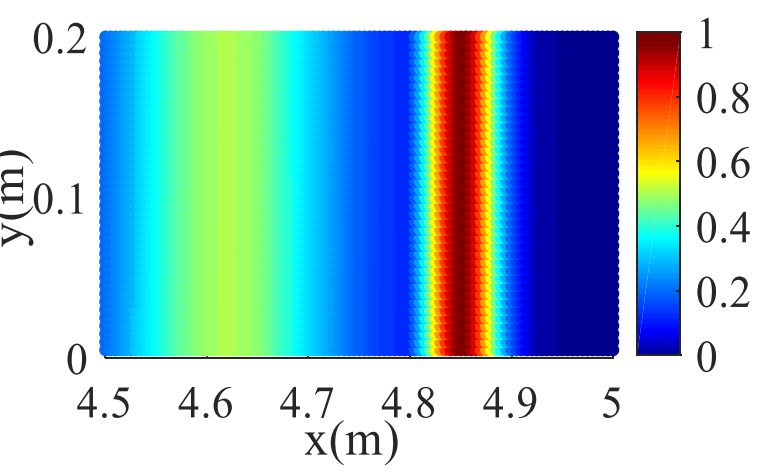

(b)

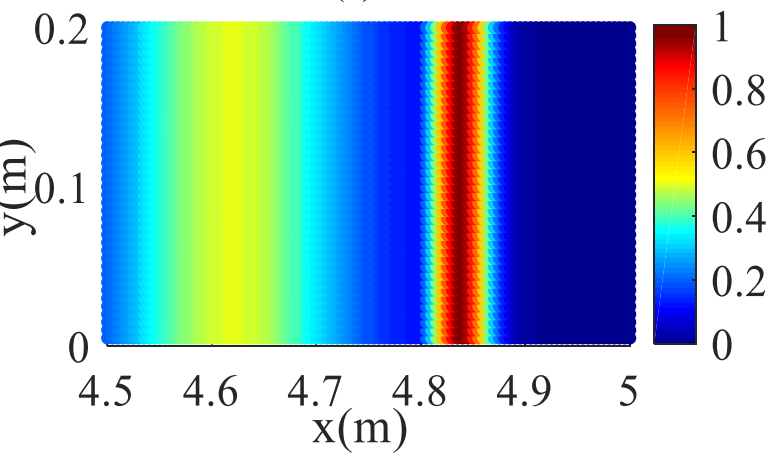

(d)

Fig. 14 The spatial distribution of sound pressure at $3.75 \mathrm{~ms}$ for (a) $10{ }^{\circ} \mathrm{C}$ (b) $20^{\circ} \mathrm{C}$ (c) $30{ }^{\circ} \mathrm{C}$ (d) $40{ }^{\circ} \mathrm{C}$ (color online)

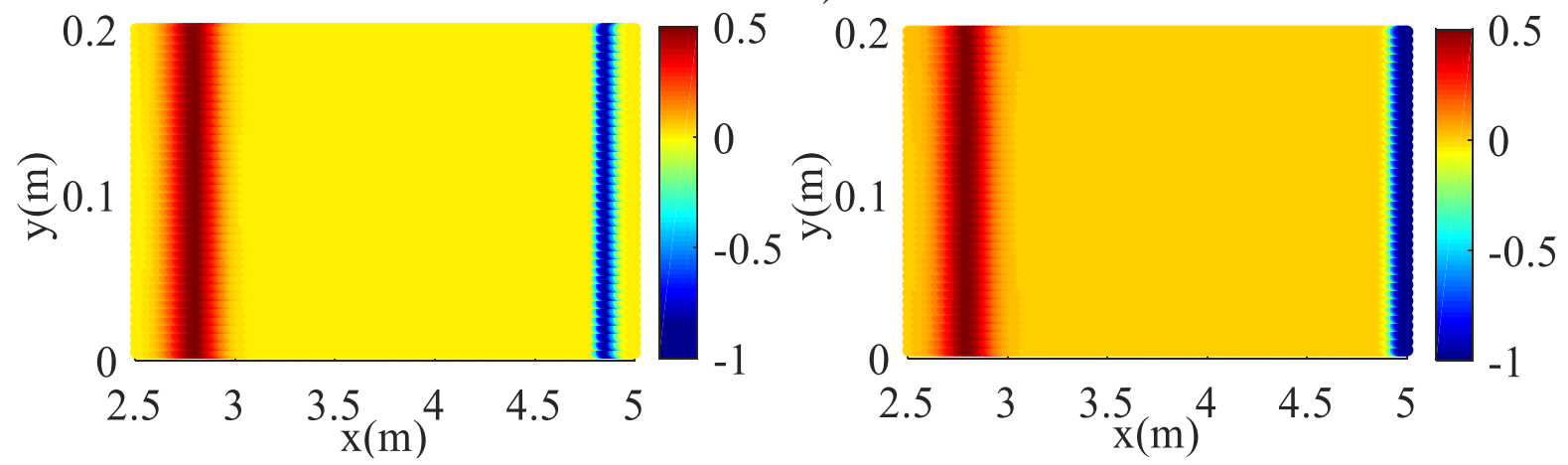

(a)

(b)

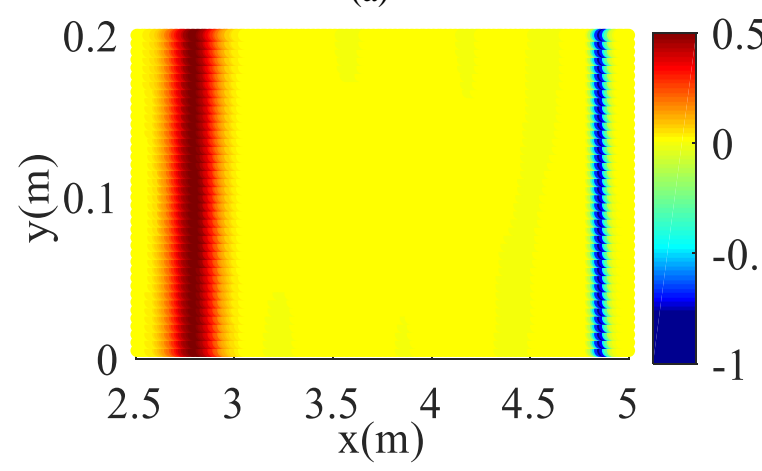

(c)

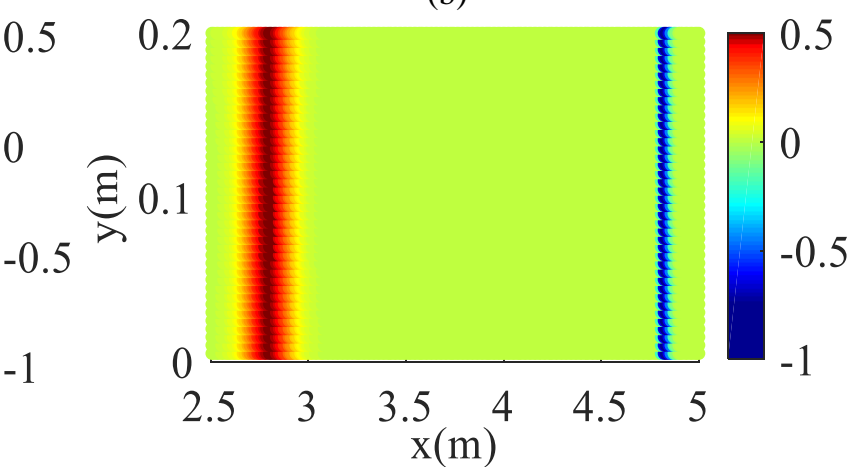

(d)

Fig. 15 The spatial distribution of sound pressure at $5.0 \mathrm{~ms}$ for (a) $10{ }^{\circ} \mathrm{C}$ (b) $20^{\circ} \mathrm{C}$ (c) $30^{\circ} \mathrm{C}$ (d) $40{ }^{\circ} \mathrm{C}$ (color online) 


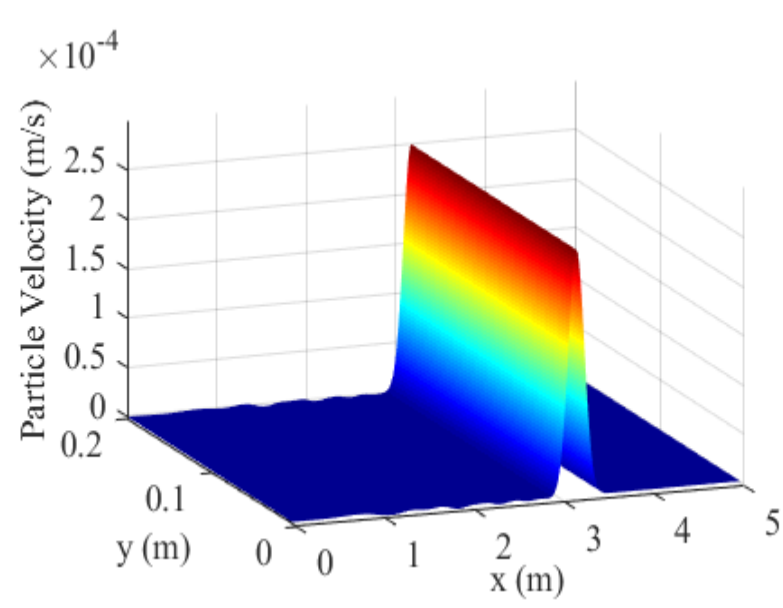

(a)

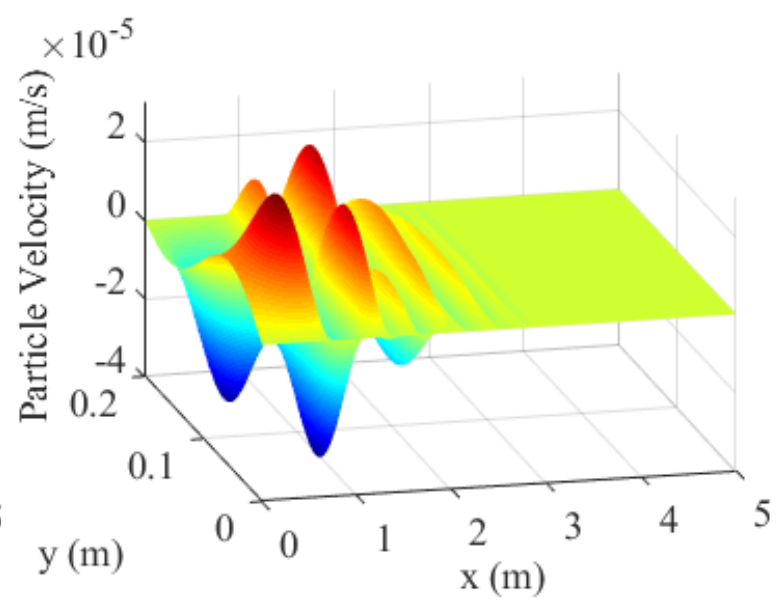

(b)

Fig. 16 The spatial distribution of particle velocities for (a) $u$ in the wave propagating direction and (b) $v$ perpendicular to the propagating direction. (color online)

Table III. Mean particle velocity for specimen B at $5.0 \mathrm{~ms}$ in different temperatures

\begin{tabular}{ccccc}
\hline Specimen B neoprene rubber & $10{ }^{\circ} \mathrm{C}$ & $20{ }^{\circ} \mathrm{C}$ & $30{ }^{\circ} \mathrm{C}$ & $40{ }^{\circ} \mathrm{C}$ \\
\hline$u$ & $2.16 \times 10^{-5} \mathrm{~m} / \mathrm{s}$ & $2.23 \times 10^{-5} \mathrm{~m} / \mathrm{s}$ & $2.30 \times 10^{-5} \mathrm{~m} / \mathrm{s}$ & $2.41 \times 10^{-5} \mathrm{~m} / \mathrm{s}$ \\
\hline$v$ & $8.67 \times 10^{-9} \mathrm{~m} / \mathrm{s}$ & $8.70 \times 10^{-9} \mathrm{~m} / \mathrm{s}$ & $8.70 \times 10^{-9} \mathrm{~m} / \mathrm{s}$ & $8.71 \times 10^{-9} \mathrm{~m} / \mathrm{s}$ \\
\hline
\end{tabular}

\section{B.3 Numerical error analysis}

To investigate the numerical error propagation of the proposed FDTD method, the relative error versus time are reported at an observation point in the centre of the $\mathrm{H}-\mathrm{N}$ domain by using ${ }^{4,5}$

$$
e_{r}(t)=20 \log \left|\frac{p_{r e f}(t)-p(t)}{p_{\text {ref }}^{\max }}\right|
$$

in which $p_{\text {ref }}(t)$ and $p_{\text {ref }}^{\max }$ represent the reference solution based on a reference sound field distribution and the relevant peak value. $p(t)$ represents the time-dependent sound pressure field of point $\mathrm{E}$ as shown in Fig. 6(b).

The relative errors for two $\mathrm{H}-\mathrm{N}$ dispersive media at different temperatures are recorded in Figs.17-18. As it can be seen from the figures, the maximum relative error appears at 1450 time steps and 2100 time steps for chloroprene rubber, and at 1800 time steps and 2600 time steps for neoprene rubber. This is due to that the only discretization error can be observed before the wave reaching the boundary. After the boundary is impacted by the incident wave, the boundary error dominates the simulation which causes the maximum relative error. Besides, it can be concluded from the figures that the relative error obtained at higher temperature are greater than that at lower temperature in trend. The reference temperature for $\mathrm{H}-\mathrm{N}$ parameter optimization is chosen at lower temperature, hence the parameters obtained at higher temperature bring the optimized error and will propagate during the FDTD simulation.

The maximum relative errors are shown in Table. IV. The maximum relative error are observed less than $-49 \mathrm{~dB}$ for both specimen simulations, proving the accuracy of the proposed FDTD method. 


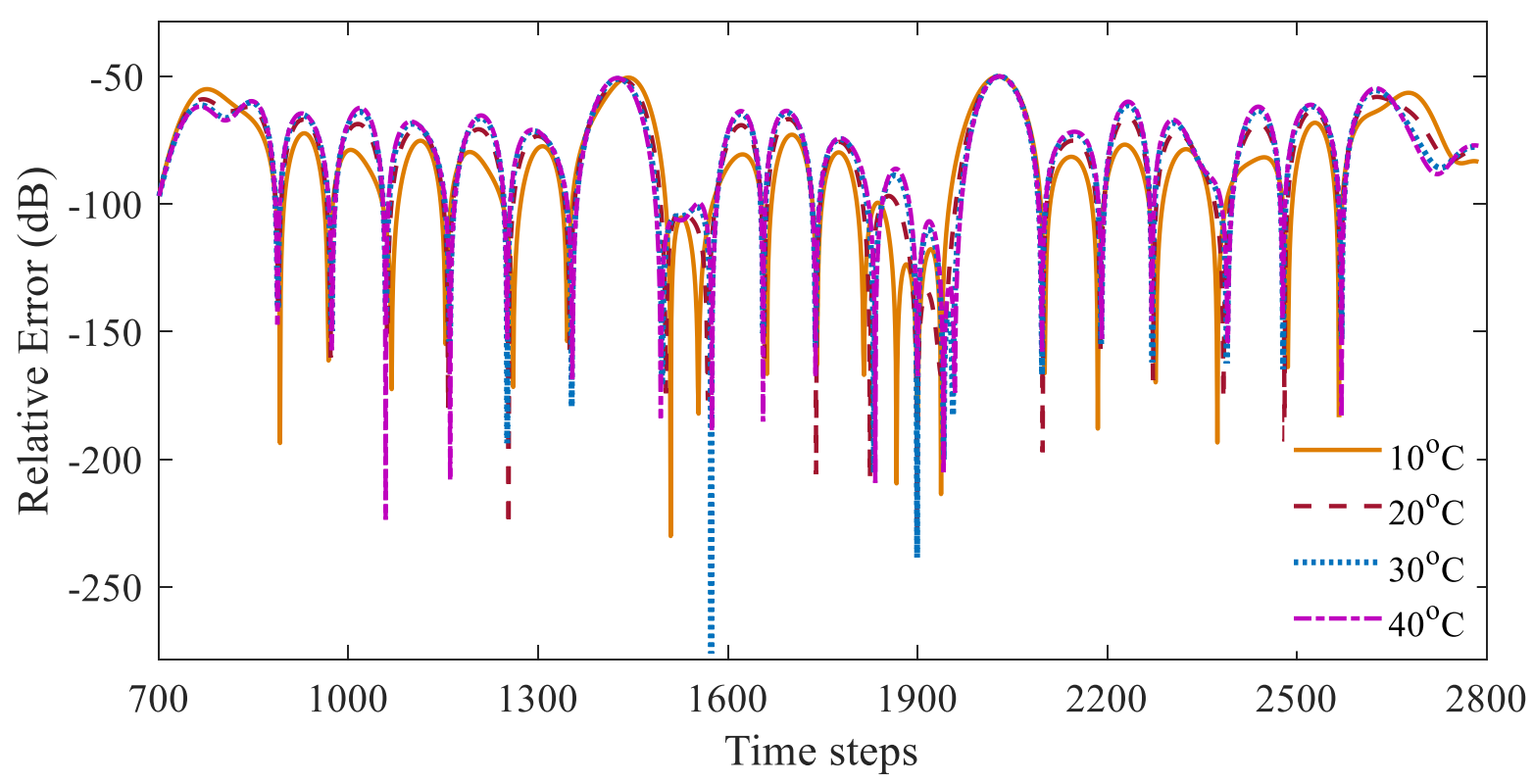

546

547

548

549

550

551

552

$\frac{\text { Table IV. Maximum relati }}{\text { Maximum relative erro }}$ (dB)

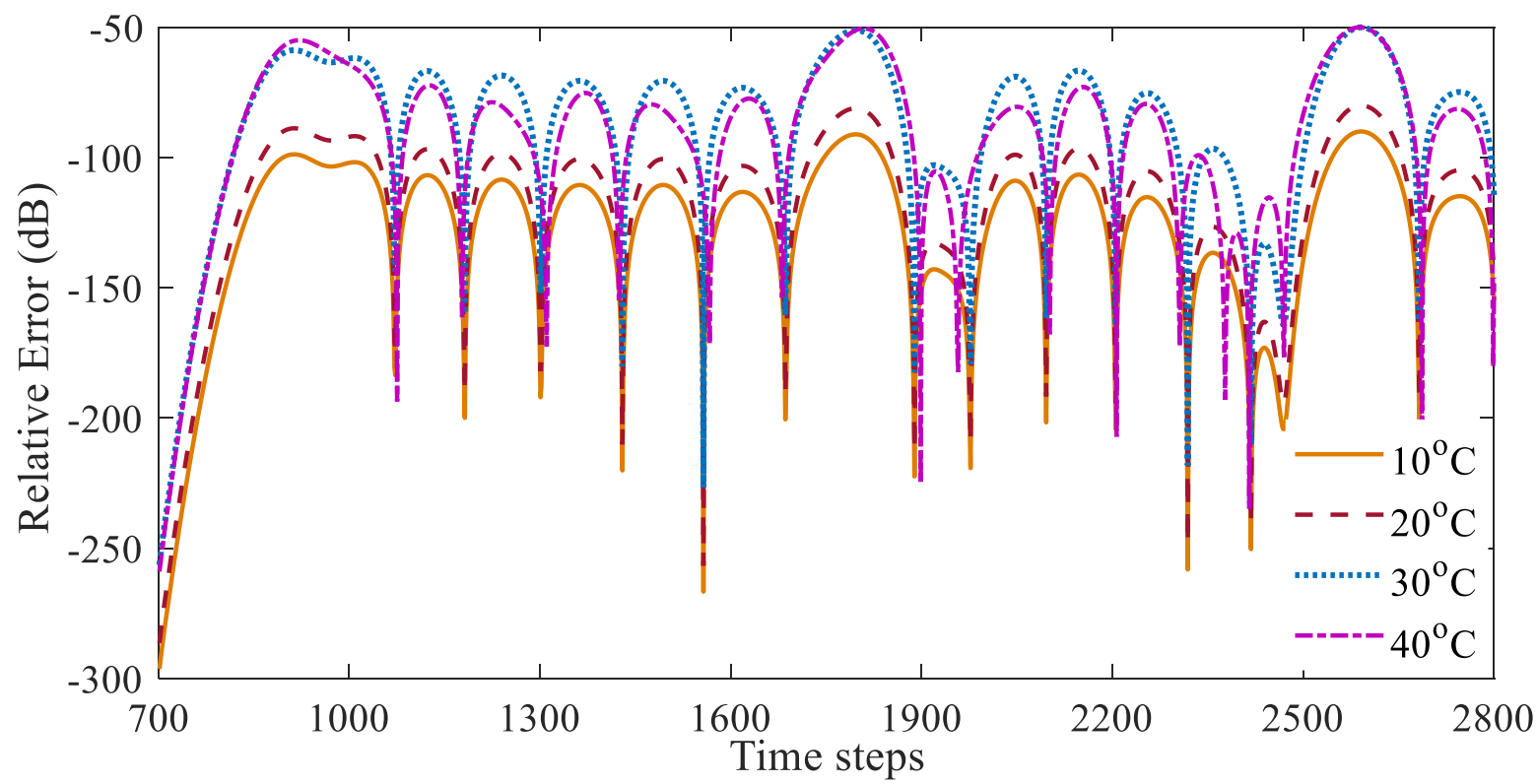

(b)

Fig. 17 Relative error of sound pressure at different temperatures for (a) chloroprene rubber (b) neoprene rubber (color online)

\begin{tabular}{ccccc}
$(\mathrm{dB})$ & $10{ }^{\circ} \mathrm{C}$ & $20{ }^{\circ} \mathrm{C}$ & $30{ }^{\circ} \mathrm{C}$ & $40{ }^{\circ} \mathrm{C}$ \\
\hline $\begin{array}{c}\text { Specimen A } \\
\text { chloroprene rubber }\end{array}$ & -50 & -50 & -49 & -49.5 \\
\hline $\begin{array}{c}\text { Specimen B } \\
\text { neoprene rubber }\end{array}$ & -83 & -76.8 & -50 & -48.8 \\
\hline
\end{tabular}




\section{Conclusion}

The main objective of this study is to develop finite difference formulation for acoustic wave propagation in elastomers within fluid field under different temperature conditions. It specially concerns the H-N mechanical response and the WLF shift function. The twodimensional FDTD formulation is expressed based on Grunwald-Letnikov fractional operator and temperature-based relaxation time. The developed 2-D FDTD scheme is applied to analyse the acoustic pulse-wave propagation in $\mathrm{H}-\mathrm{N}$ dispersive medium contained in the fluid field. Acoustic absorptions for different elastomers have been calculated by using the proposed FDTD formulation and compared with experiment data. Numerical results for 2-D spatial distribution of sound pressure and particle velocities demonstrates the feasibility of the proposed FDTD method in the study of broadband wave propagation in $\mathrm{H}-\mathrm{N}$ dispersive media.

\section{ACKNOWLEGMENT}

This work was financially supported by the National Key Research and Development Program of China (Grant No. 2016YFF0200902), the National Natural Science Foundation of China (Grants Nos. 11474230 and 11704314) and the International Cooperation Training Program for the Innovative Talents of China Scholarship Council.

\section{APPENDIX A}

By using Eqs. (8a) and (9), the relation between loss factor and the storage modulus can be represented by the Wicket function. ${ }^{40}$ Since the Wicket function remains the same for different relaxation times, ${ }^{41,42}$ it can be adopted to determine $\mathrm{H}-\mathrm{N}$ parameters $E_{0}, E_{\infty}, \alpha$ and $\beta$.

In the DMTA experiments, loss modulus, $E^{\prime \prime}$, and the loss factor, $\tan \delta$, is measured as a function of frequency. The calculated frequency-dependent complex modulus is also obtained by using initial values for $\mathrm{H}-\mathrm{N}$ parameters $E_{0}, E_{\infty}, \alpha, \beta$ with $\tau=1$. The optimized values of H-N parameters can be determined based on Levenberg-Marquardt method by using the MATLAB optimization toolbox.

According to Levenberg-Marquardt, the $\mathrm{H}-\mathrm{N}$ parameters are updated in specified ranges at each iteration as $10^{-4}$ for parameters $\alpha, \beta$ and $10^{2}$ for parameters $E_{0}, E_{\infty}$. The convergence criteria are specified based on the Wicket and Cole-Cole formulas as:

$$
\begin{gathered}
F_{1}=\frac{\sum_{f}\left|E_{\text {mea }}^{\prime \prime}-E_{\text {cal }}^{\prime \prime}\right|^{2}}{\sum_{f}\left|E_{\text {mea }}^{\prime \prime}\right|^{2}} \\
F_{2}=\frac{\sum_{f}\left|\log \left(\tan \delta_{\text {mea }}\right)-\log \left(\tan \delta_{\text {cal }}\right)\right|^{2}}{\sum_{f}\left|\log \left(\tan \delta_{\text {mea }}\right)\right|^{2}}
\end{gathered}
$$


588

589

590

591

592

593

594

595

596

597

598

599

600

601

602

603

604

605

606

607

608

609

610

611

612

613

614

615

616

617

618

in which $F_{1}$ represents the Wicket error formula and $F_{2}$ represents the Cole-Cole error formula ${ }^{41}$. $E_{\text {mea }}^{\prime \prime}$ and $E_{c a l}^{\prime \prime}$ represent the measured and calculated loss modulus, respectively. $\tan \delta_{\text {mea }}$ and $\tan \delta_{\text {cal }}$ represent the measured and calculated loss factor, respectively. The convergence tolerances are specified as $F_{1}=100$ and $F_{2}=0.0001$.

The procedure for Levenberg-Marquardt method can be summarized as follow:

(1) Initialize $\mathrm{H}-\mathrm{N}$ parameters as $E_{0}=3 \times 10^{7} \mathrm{~Pa}, E_{\infty}=3 \times 10^{9} \mathrm{~Pa}, \alpha=0.5, \beta=0.5$ at $\tau=1$ for a frequency range of $10^{7} \mathrm{~Hz}<\omega<5 \times 10^{9} \mathrm{~Hz}$

(2) Obtain complex modulus $E^{*}(\omega)$, storage modulus $E^{\prime}$, loss modulus $E^{\prime \prime}$ and loss factor $\tan \delta$ from Eqs. (6), (8a), (8b) and (9) for a given frequency range.

(3) Calculate $F_{1}$ and $F_{2}$ by using Eqs. (A.1) and (A.2).

(4) Check if $F_{1}<10^{-2}$ and $F_{2}<10^{-4}$. If true, output the optimized value for H-N parameters $E_{0}, E_{\infty}, \alpha$ and $\beta$. If false, estimate new values for $E_{0}, E_{\infty}, \alpha, \beta$ and $\tau=1$ by using the specified iteration interval for a given frequency range.

After finding the H-N parameters, the expression for relaxation time, $\tau$ can be calculated by using Eq. (1) for given complex Young's modulus $E^{*}(\omega)$ as ${ }^{41}$

$$
\tau=-i \exp \left\{\log \left[\exp \left(\log \left(\frac{E_{0}-E_{\infty}}{E^{*}-E_{\infty}}\right) / \beta\right)-1\right] / \alpha \omega\right\}
$$

\section{APPENDIX B}

The sound pressure at the spatial point $(x, y)$ can be considered with the assumption of amplitude equals to 1 as

$$
p_{0}(x, y, t)=e^{j k_{x} x} e^{j k_{y} y} e^{-j \omega t}
$$

in which $k_{x}, k_{y}$ represent the $\mathrm{x}$ - and $\mathrm{y}$-component of the wave number $k$. The particle velocities $u, v$ and sound pressure $p$ of $\mathrm{H}-\mathrm{N}$ media at the specific spatial point can be expressed as ${ }^{44}$

1

$$
\begin{aligned}
& \left.u\right|_{i+1 / 2, j} ^{n}=u_{0} Z^{n} \cdot e^{j k_{x}(i+1 / 2) h} e^{j k_{y}(j h)} \\
& \left.v\right|_{i, j+1 / 2} ^{n}=v_{0} Z^{n} \cdot e^{j k_{x}(i h)} e^{j k_{y}(j+1 / 2) h} \\
& \left.p\right|_{i, j} ^{n+1 / 2}=p_{0} Z^{n+1 / 2} \cdot e^{j k_{x}(i h)} e^{j k_{y}(j h)}
\end{aligned}
$$

where $u_{0}, v_{0}, p_{0}$ represent the initial values and $Z$ represents the complex amplification ratio per time step.

Substituting Eqs. (B.2a)-(B.2c) into the FDTD formulas of constitutive relation for H-N media yields

$$
u_{0} Z^{n+1} e^{j k_{x}(i+1 / 2) h} e^{j k_{y}(j h)}=u_{0} Z^{n} e^{j k_{x}(i+1 / 2) h} e^{j k_{y}(j h)}+\frac{\Delta t}{\rho_{s} h}\left(p_{0} Z^{n+1 / 2} e^{j k_{x}(i+1) h} e^{j k_{y}(j h)}-p_{0} Z^{n+1 / 2} e^{j k_{x}(i h)} e^{j k_{y}(j h)}\right)
$$




$$
\begin{aligned}
v_{0} Z^{n+1} e^{j k_{x}(i h)} e^{j k_{y}(j+1 / 2) h} & =v_{0} Z^{n} e^{j k_{x}(i h)} e^{j k_{y}(j+1 / 2) h}+\frac{\Delta t}{\rho_{s} h}\left(p_{0} Z^{n+1 / 2} e^{j k_{x}(i h)} e^{j k_{y}(j+1) h}-p_{0} Z^{n+1 / 2} e^{j k_{x}(i h)} e^{j k_{y}(j h)}\right) \\
p_{0} Z^{n+1 / 2} e^{j k_{x}(i h)} e^{j k_{y}(j h)} & =p_{0} Z^{n-1 / 2} e^{j k_{x}(i h)} e^{j k_{y}(j h)}+\frac{\Delta t}{h}\left[C_{1111}\left(u_{0} Z^{n} e^{j k_{x}(i+1 / 2) h} e^{j k_{y}(j h)}-u_{0} Z^{n} e^{j k_{x}(i-1 / 2) h} e^{j k_{y}(j h)}\right)\right. \\
& \left.++C_{1122}\left(v_{0} Z^{n} e^{j k_{x}(i h)} e^{j k_{y}(j+1 / 2) h}-v_{0} Z^{n} e^{j k_{x}(i h)} e^{j k_{y}(j-1 / 2) h}\right)\right]
\end{aligned}
$$

621 in which $h=\Delta x=\Delta y$ represents the discrete spatial interval.

622

623

624

625

626

627

628

629

630

631

632

633

634

635

$$
\left(Z^{n+1 / 2}-Z^{n-1 / 2}\right)^{3}-\frac{C_{1111}(\Delta t)^{2}}{\rho_{s} h^{2}} \cdot 2\left(j \sin \frac{k_{x} h}{2}\right)^{2}-\frac{C_{1122}(\Delta t)^{2}}{\rho_{s} h^{2}} \cdot 2\left(j \sin \frac{k_{y} h}{2}\right)^{2}=0
$$

and can be simplified as

$$
Z^{2}-2 A Z+1=0
$$

with $A=1-2\left(\frac{C_{1111}(\Delta t)^{2}}{\rho_{s} h^{2}} \sin ^{2} \frac{k_{x} h}{2}+\frac{C_{1122}(\Delta t)^{2}}{\rho_{s} h^{2}} \sin ^{2} \frac{k_{y} h}{2}\right)$. A solution of the Eq. (B.6b) is

$$
Z=\left\{\begin{array}{c}
A \pm \sqrt{A^{2}-1}(A<-1) \\
A \pm j \sqrt{1-A^{2}}(-1<A<1)
\end{array}\right.
$$

636

637

638

The time domain convergency requires the absolute value of $Z$ greater than 1 , hence $-1 \leq A \leq 1$ becomes the critical condition for the stability which yields

$$
\frac{C_{1111}(\Delta t)^{2}}{\rho_{s} h^{2}} \sin ^{2} \frac{k_{x} h}{2}+\frac{C_{1122}(\Delta t)^{2}}{\rho_{s} h^{2}} \sin ^{2} \frac{k_{y} h}{2}<1
$$


Since $\sin \left(k_{x} h / 2\right) \leq 1$ and $\sin \left(k_{y} h / 2\right) \leq 1$ for arbitrary $k$, the Courant-Friedrichs-Lewy condition for H-N media can be obtained by using Eq. (B.8b)

$$
\sqrt{\frac{C_{1111}+C_{1122}}{\rho_{s}}} \frac{\Delta t}{\Delta x} \leq 1
$$

Similarly, the Courant-Friedrichs-Lewy condition for fluid flow can be obtained by ignoring the infinitesimal viscosity resistance as

$$
\sqrt{\frac{K_{F}}{\rho_{F}}} \frac{\Delta t}{\Delta x} \leq C_{\max }
$$

\section{REFERENCES}

${ }^{1}$ J. H. Zhu, Y. S. Wang, Q. Z. Wen and S. R. Yao, "The development and application of underwater acoustic absorption polymer materials," Polymer Materials Science and Engineering. 21, 46(2005).

${ }^{2}$ L. Sun, H. Hou, "Transmission loss measurement of acoustic material using time-domain pulse-separation method," The Journal of the Acoustical Society of America. 129(4), 1681-1684(2011).

${ }^{3}$ A. W. Nolle, "Acoustic Determination of the Physical Constants of Rubber-Like Materials," The Journal of the Acoustical Society of America. 19, 194-201(1947).

${ }^{4}$ P. Bia, D. Caratelli, L. Mescia, R. Cicchetti, G. Maione and F. Prudenzano, "A novel FDTD formulation based on fractional derivatives for dispersive Havriliak-Negami media." Signal Processing. 107, 312-318(2015).

${ }^{5}$ L. Mescia, P. Bia, D. Caratelli, "Fractional derivative based FDTD modeling of transient wave propagation in Havriliak-Negami media," IEEE Transactions on Microwave Theory and Techniques. 62(9), 1920-1929(2014). ${ }^{6}$ A. A. Khamzin, R. R. Nigmatullin and I. I. Popov, "Microscopic model of a non-Debye dielectric relaxation: The Cole-Cole law and its generalization," Theoretical and Mathematical Physics. 173(2), 1604-1619(2012).

${ }^{7}$ A. A. Khamzin, R. R. Nigmatullin and I. I. Popov, "Log-periodic corrections to the Cole-Cole expression in dielectric relaxation," Physica A: Statistical Mechanics and its Applications. 392(1), 136-148(2013).

${ }^{8}$ R. R. Nigmatullin, Y. E. Ryabov, "Cole-Davidson dielectric relaxation as a self-similar relaxation process," Physics of the Solid State. 39(1), 87-90(1997).

${ }^{9}$ R. T. Sibatov, V. V. Uchaikin and D. V. Uchaikin, "Fractional wave equation for dielectric medium with Havriliak-Negami response," In Fractional Dynamics and Control. Springer, New York, NY, 293-301(2012).

${ }^{10} \mathrm{~V}$. V. Uchaikin and R. Sibatov, "Fractional kinetics in solids: anomalous charge transport in semiconductors, dielectrics, and nanosystems," World Scientific (2011).

${ }^{11}$ W. M. Madigosky, G. F. Lee and J. M. Niemiec, "A method for modeling polymer viscoelastic data and the temperature shift function," The Journal of the Acoustical Society of America. 119, 3760-3765(2006).

${ }^{12} \mathrm{R}$. W Chan, "Estimation of viscoelastic shear properties of vocal-fold tissues based on time-temperature superposition," The Journal of the Acoustical Society of America. 110, 1548-1561(2001).

${ }^{13}$ C. S. Antonopoulos, N. V. Kantartzis, I. T. Rekanos, "FDTD method for wave propagation in HavriliakNegami media based on fractional derivative approximation," IEEE Transactions on Magnetics. 53(6), 1-4(2017) ${ }^{14} \mathrm{~J}$. Zhao, M. Bao, X. Wang, H. Lee and S. Sakamoto, "An equivalent fluid model based finite-difference timedomain algorithm for sound propagation in porous material with rigid frame," The Journal of the Acoustical Society of America. 143(1), 130-138(2018).

${ }^{15}$ J. Zhao, Z. Chen, M. Bao, H. Lee and S. Sakamoto, "Two-dimensional finite-difference time-domain analysis of sound propagation in rigid-frame porous material based on equivalent fluid model," Applied Acoustics. 146, 204-212(2019). 
${ }^{16} \mathrm{H}$. Suzuki, A. Omoto and K. Fujiwara, "Treatment of boundary conditions by finite difference time domain method," Acoustical Science and Technology. 28(1), 16-26(2007).

$682{ }^{17} \mathrm{~S}$. Su, W. Dai, D. T. Haynie and N. Simicevic, "Use of the z-transform to investigate nanopulse penetration of 683 biological matter," Bioelectromagnetics. 26(5), 389-397(2005).

$684{ }^{18}$ B. Guo, J. Li and H. Zmuda, "A new FDTD formulation for wave propagation in biological media with Cole685 Cole model,” IEEE Microwave and Wireless Components Letters. 16(12), 633-635(2006).

$686{ }^{19}$ W. J. Chen, W. Shao and B. Z. Wang, "ADE-Laguerre-FDTD method for wave propagation in general 687 dispersive materials," IEEE Microwave and Wireless Components Letters. 23(5), 228-230(2013).

$688{ }^{20}$ I. T. Rekanos and T. G. Papadopoulos, "FDTD modeling of wave propagation in Cole-Cole media with 689 multiple relaxation times," IEEE Antennas and Wireless Propagation Letters, 9, 67-69(2010).

$690{ }^{21}$ F. Torres, P. Vaudon and B. Jecko, "Application of fractional derivatives to the FDTD modeling of pulse 691 propagation in a Cole-Cole dispersive medium," Microwave and Optical Technology Letters. 13(5), 300692 304(1996).

$693{ }^{22}$ F. Iijima, T. Tsuchiya and N. Endoh, "Analysis of characteristics of underwater sound propagation in the 694 ocean by a finite difference time domain method," Japanese Journal of Applied Physics. 39(5S), 3200(2000).

$695{ }^{23}$ I. M. Hallaj and R. O. Cleveland, "FDTD simulation of finite-amplitude pressure and temperature fields for 696 biomedical ultrasound," The Journal of the Acoustical Society of America. 105(5), L7-L12(1999).

$697{ }^{24}$ F. D. Hastings, J. B. Schneider, S. L. Broschat and E. I. Thorsos, “An FDTD method for analysis of scattering 698 from rough fluid-fluid interfaces,” IEEE Journal of Oceanic Engineering. 26(1), 94-101(2001).

$699{ }^{25}$ W. C. Chew and Q. H. Liu, "Perfectly matched layers for elastodynamics: a new absorbing boundary 700 condition," Journal of Computational Acoustics. 4(04), 341-359(1996).

$701{ }^{26} \mathrm{Q}$. H. Liu. "Perfectly matched layers for elastic waves in cylindrical and spherical coordinates," The Journal of 702 the Acoustical Society of America. 105(4), 2075-2084(1999).

$703{ }^{27}$ H. Schiessel, R. Metzler, A. Blumen, and T. F. Nonnenmacher, "Generalized viscoelastic models: their 704 fractional equations with solutions," Journal of Physics A: Mathematical and General. 28, 6567(1995).

$705{ }^{28}$ L. Dagdug and L. S. García-Colín, "Generalization of the Williams-Landel-Ferry equation," Physica A: 706 Statistical Mechanics and its Applications. 250, 133-141(1998).

$707{ }^{29}$ A. A. A. Kilbas and H. M. Srivastava and J. J. Trujillo, "Partial fractional differential equations," In Theory and applications of fractional differential equations. Elsevier Science Limited, 347-391(2006).

${ }^{30} \mathrm{~V}$. V. Uchaikin. Fractional derivatives for physicists and engineers. Springer, Berlin, 2013.

${ }^{31}$ R. Garrappa, "A Grünwald-Letnikov scheme for fractional operators of Havriliak-Negami type," Recent Advances in Applied Mathematics, Modelling and Simulation. 34, 70-76(2014).

${ }^{32}$ K. S. Yee and J. S. Chen, "The finite-difference time-domain (FDTD) and the finite-volume time-domain (FVTD) methods in solving Maxwell's equations," IEEE Transactions on Antennas and Propagation. 45(3), 354363(1997).

${ }^{33}$ J. Lubbers and R. Graaff, "A simple and accurate formula for the sound velocity in water," Ultrasound in Medicine and Biology. 24(7), 1065-1068(1998).

${ }^{34}$ Y. Zhang, X. Li and T. Zhang, "Modeling sound propagation using the corrective smoothed particle method with an acoustic boundary treatment technique," Mathematical and Computational Applications. 22(1), 26(2017).

${ }^{35}$ H. Assi, R. S. C. Cobbold, "A perfectly matched layer formulation for modeling transient wave propagation in an unbounded fluid-solid medium," The Journal of the Acoustical Society of America. 139(4), 15281536(2016).

${ }^{36} \mathrm{H}$, Chang, and C. J. Randall, "Finite-difference time-domain modeling of elastic wave propagation in the cylindrical coordinate system," IEEE 1988 Ultrasonics Symposium Proceedings. 397-402(1988).

${ }^{37}$ Y. Huang, H. Hou, S. Oterkus, Z. Wei, and Zhang. S, "Mechanical and acoustic performance prediction model for elastomers in different environmental conditions," The Journal of the Acoustical Society of America. 144(4), 2269-2280(2018).

${ }^{38}$ J. R. Fincke, W. D. Swank and C. L. Jeffery, "Simultaneous measurement of particle size, velocity, and temperature in thermal plasmas," IEEE Transactions on Plasma Science. 18(6), 948-957(1990). 
${ }^{39} \mathrm{~S}$. Li, Z. Y and A. J. Jaworski, "Application of laser-based instrumentation for measurement of time-resolved temperature and velocity fields in the thermoacoustic system," International Journal of Thermal Sciences. 49(9), 1688-1701(2010).

733 40"Damping materials-Graphical presentation of the complex modulus." ISO, 10112(1991).

$734{ }^{41}$ T. T. Kararli, J. B. Hurlbut and T. E. Needham, "Glass-rubber transitions of cellulosic polymers by dynamic 735 mechanical analysis," Journal of Pharmaceutical Sciences. 79, 845-848(1990).

$736{ }^{42}$ W. M. Madigosky, G. F. Lee and J. M. Niemiec, "A method for modeling polymer viscoelastic data and the 737 temperature shift function," The Journal of the Acoustical Society of America. 119, 3760-3765(2006).

738 43J. D. Ferry, "Viscoelastic properties of polymers," New York: Wiley, 1980.Chapt.15, pp.361.

$739{ }^{44}$ T. Sakuma, S. Sakamoto and T. Otsuru, "Computational simulation in architectural and environmental 740 acoustics," Springer, 2014. Chapt.2, pp.30. 